\title{
New Algorithm and Processor for Obtaining Maritime Information from Sentinel-1 Radar Imagery for Near Real Time Services
}

Andrey Pleskachevsky, Sven Jacobsen, Björn Tings Egbert Schwarz, Detmar Krause, Holger Daedelow
DLR, Maritime Safety and Security Lab Bremen

DLR, National Ground Segment, Neustrelitz

- Examples and concept

- Background

- Model Functions Tuning

- NRT implementation

- Outlook

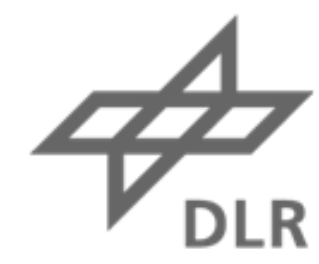




\section{Short description}

The new empirical algorithm allows estimation of total integrated sea state parameters and also partial integrated parameters including

- significant wave height $H_{s}$,

- first moment wave period $T_{m 1}$,

- second moment period $T_{m 2}$,

- mean period $T_{m}$

- like swell (dominant and secondary) and windsea wave heights $S_{w 1}, S_{w 2}, S_{w w}$

- windsea period $T_{w}$.

The algorithm allows processing of different S1 Synthetic Aperture Radar (SAR) modes with different resolution into sea state fields:

- For Sentinel-1 S1 Wave Mode (WV), acquires multiple vignettes with an extent of $\sim 20 \mathrm{~km} \times 20 \mathrm{~km}$ and each displaced by $100 \mathrm{~km}$ along satellite tracks in open ocean (global) with relatively high spatial resolution of $\sim 4 \mathrm{~m}$ wave height can be estimated with accuracy of $\sim 35 \mathrm{~cm}$. This is comparable with the accuracy of satellite altimetry and a new achievement for SAR based techniques.

- For Sentinel-1 Interferometric Wide Swath Mode (IW) covers area-strips of thousand kilometres of earth and ocean surface in coastal areas with a resolution of $\sim 20 \mathrm{~m}$ by sequences of multiple images with an approximate size of $200 \mathrm{~km} \times 250 \mathrm{~km}$ the accuracy of $\sim 70 \mathrm{~cm}$

The algorithm has been integrated into a prototype processor for Sentinel-1 SAR imagery. The DLR Ground Station Neustrelitz applies this prototype as part of a near real-time demonstrator MSA service. 
1. Concept and Examples

2. Background

3. Model Functions, Tuning

4. NRT implementation

5. Outllook 


\section{Sentinel-1A - 2014}

Sentinel-1B - 2016

flight $704 \mathrm{~km}$

- ground speed $6.8 \mathrm{~km} / \mathrm{s}$

C-Band Radar with wavelength of $5.6 \mathrm{~cm}$

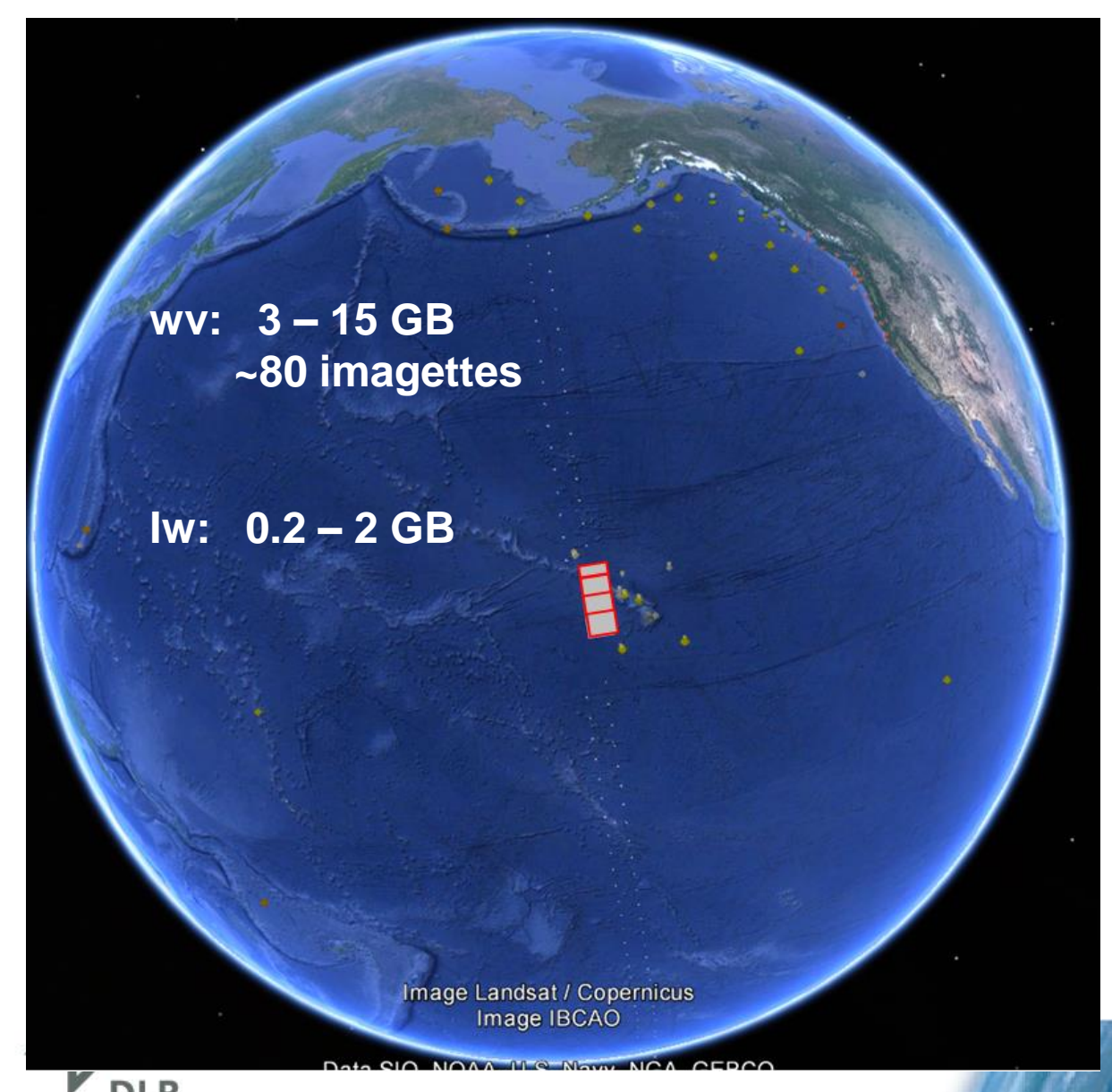

DLR
- IW - Interferometric Wide Swath Mode - Coastal areas

$\sim 200 \mathrm{~km} \times 250 \mathrm{~km}, \sim 35 \mathrm{~m}$ resolution, $10 \mathrm{~m}$ pixel

- WV - Wave Mode - Ocean

$\sim 20 \mathrm{~km} \times 20 \mathrm{~km}$ vignette each $100 \mathrm{~km}, \sim 5 \mathrm{~m}$ pixel

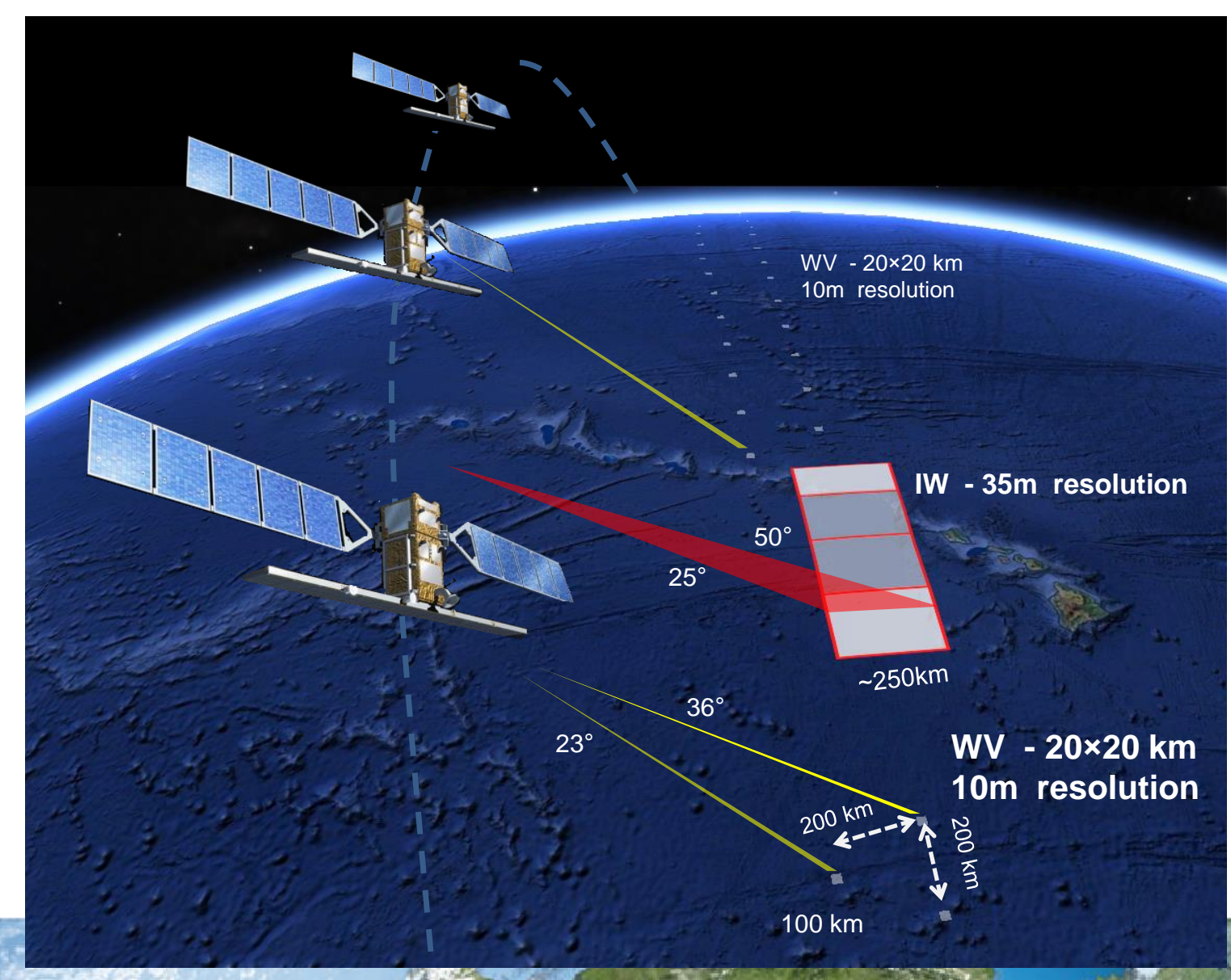




\section{Integrated Processor for MSA: Near Real Time services(NRT)}

DLR Maritime safety and security Lab Bremen algorithms and processor development

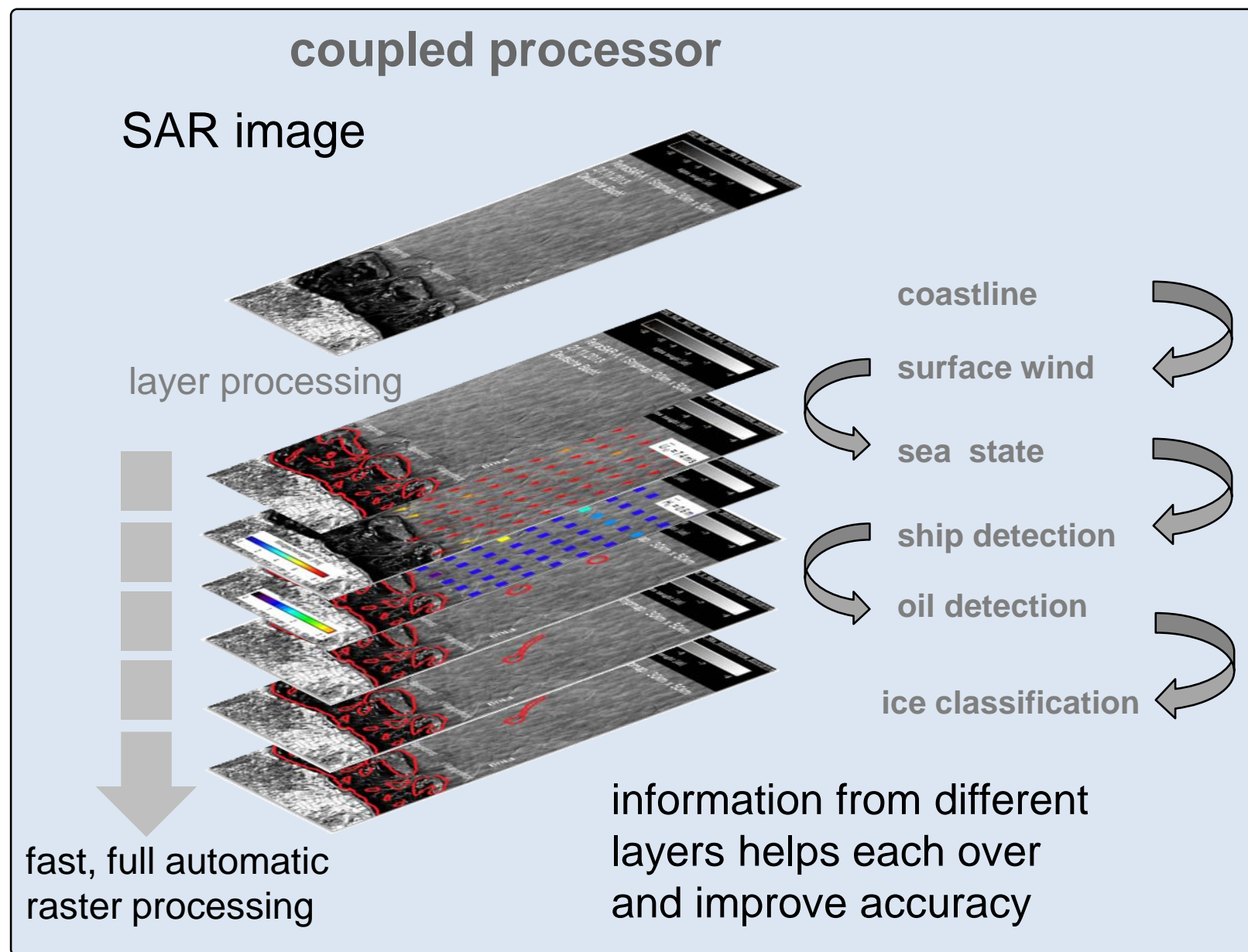

DLR Ground Station Neustrelitz (NZ) NRT chain
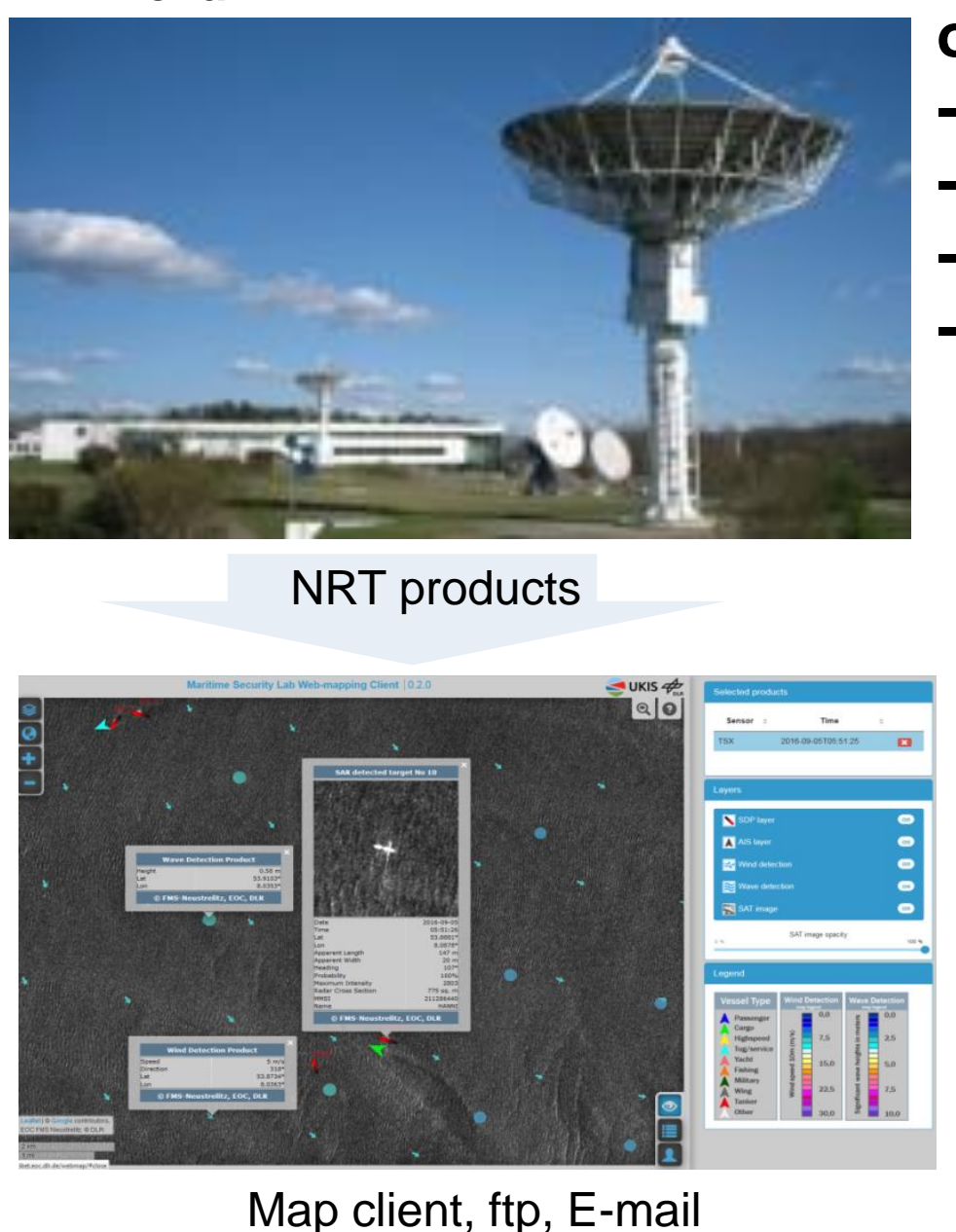

operationally: -sea state -wind -ships -icebergs

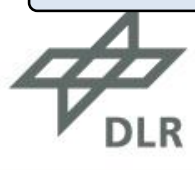

FUSION

WITH DATA

FROM OTHER SOURCES

+ measurements

+ forecast

+ ship AIS 


\section{NRT services: SENTINEL-1 waves, wind, ships}

\section{Raster: $6 \mathrm{~km}$, Subscenes: $2.5 \mathrm{~km} \times 2.5 \mathrm{~km}$}

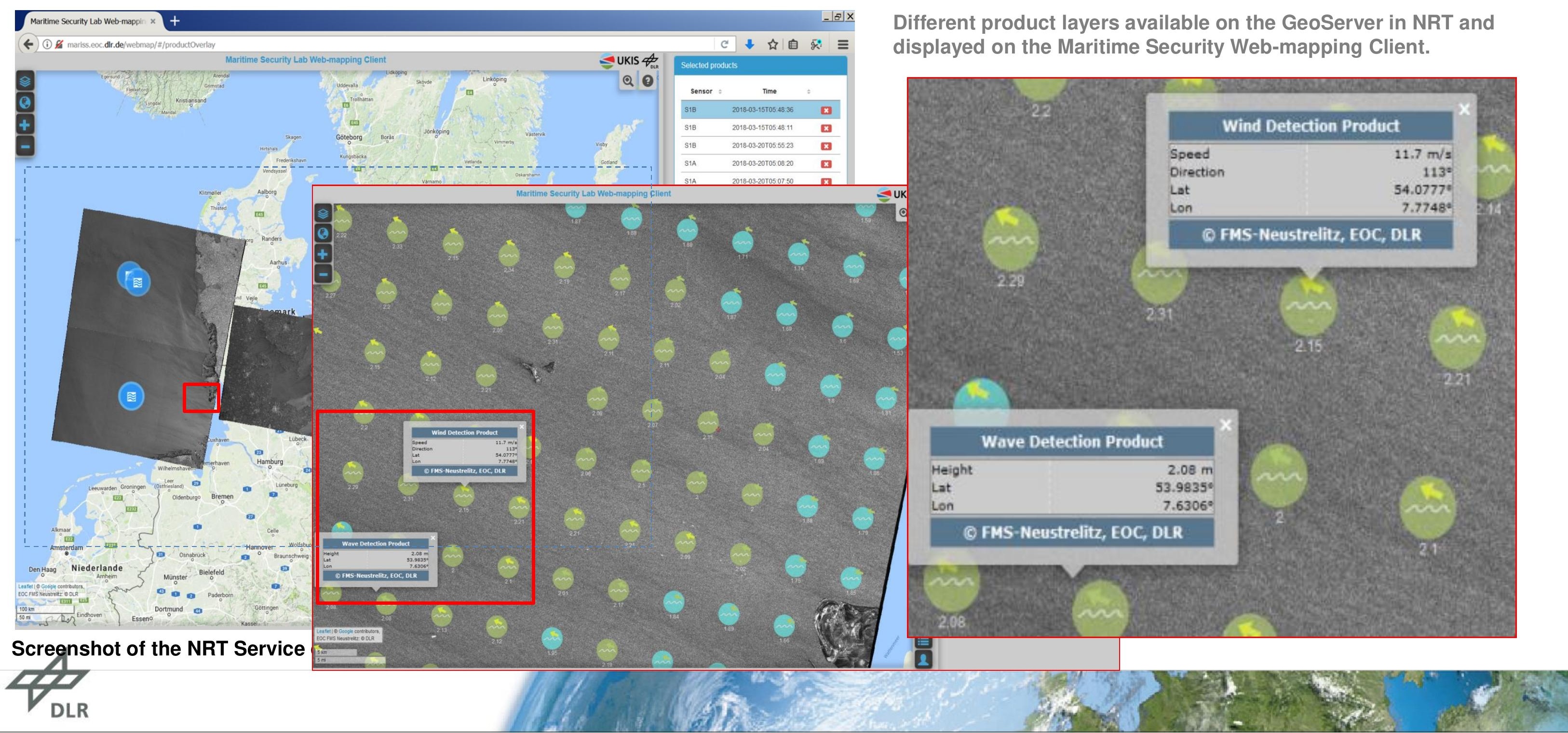




\section{Hurricane „Irma“ 2017 (S-1)}

SENTINEL S-1 IV VV 2017-09-09 23:33 UTC TOTAL SIGNIFICANT WAVE HEIGHT

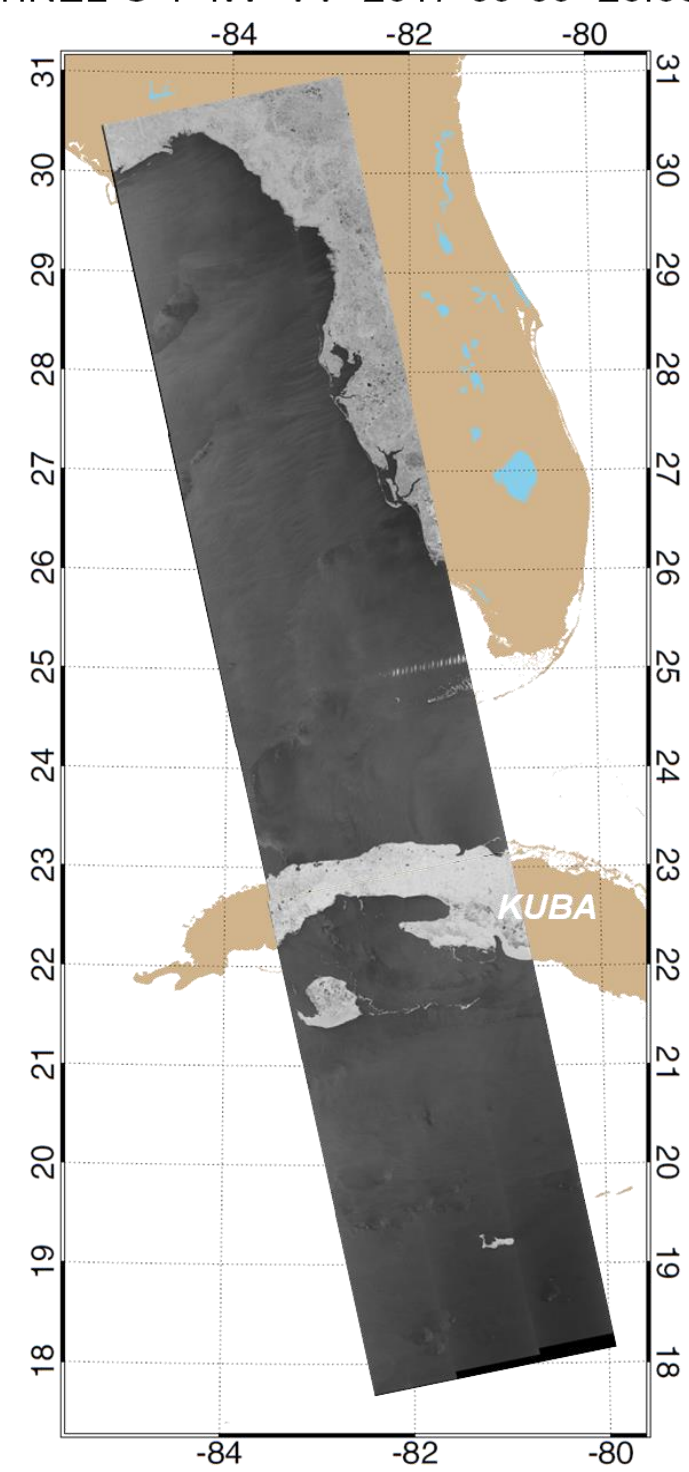

บLn

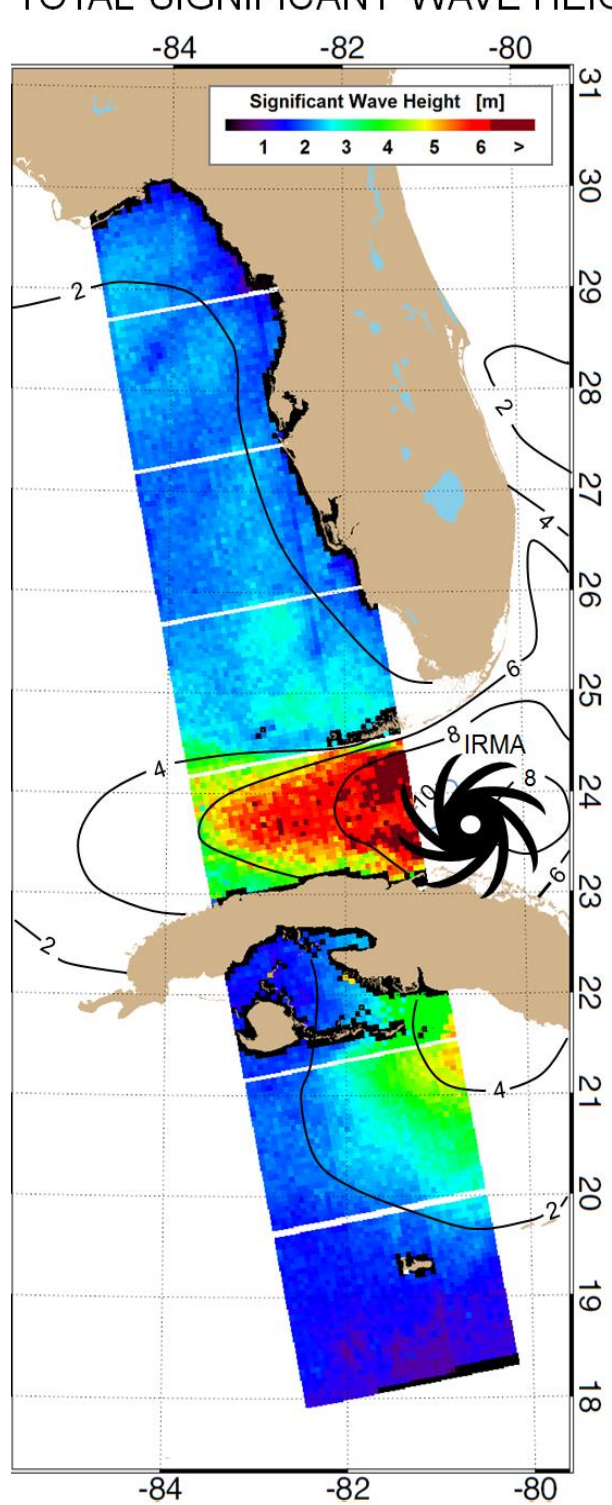

new techniques and algorithms allow observation and validation of forecast models worldwide

2017-09-10 23:25 UTC

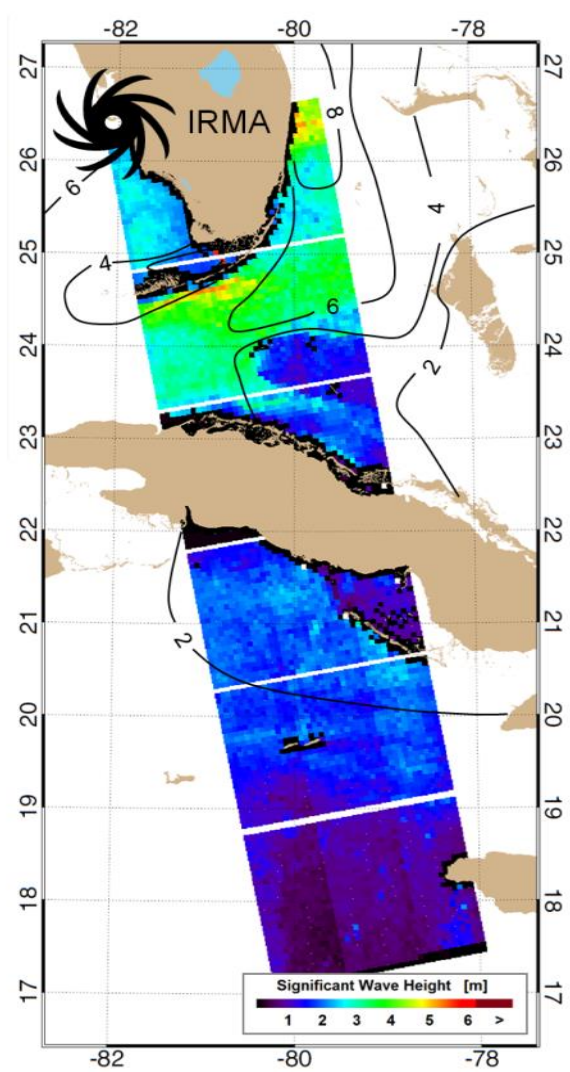

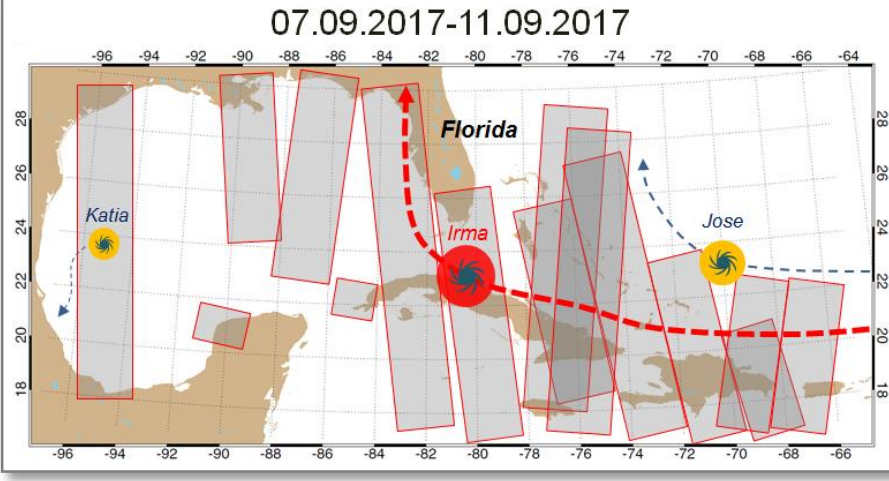

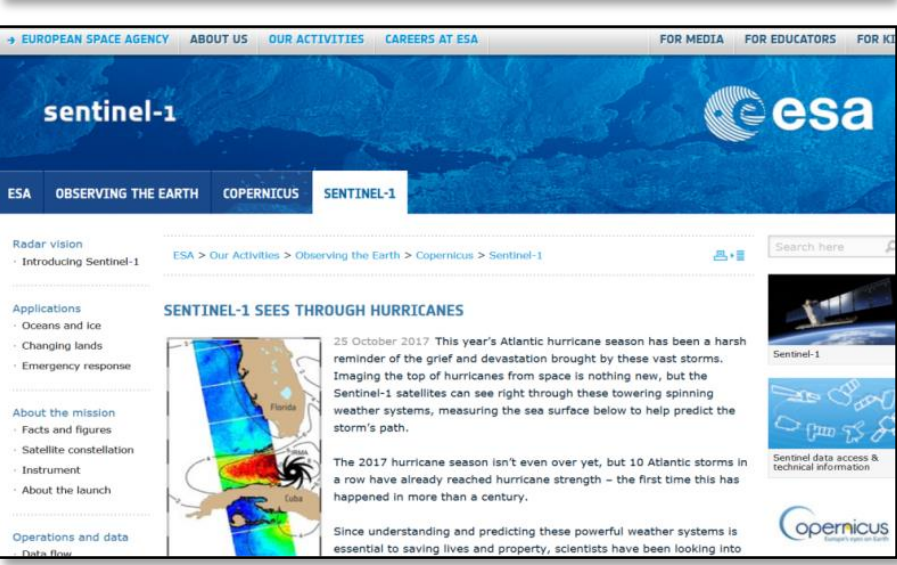

ESA news:

Sentinel-1 sees through hurricanes

"... information about the sea state can help to assess how destructive a hurricane is and can predict its path respectively time and location on which it will make landfall ...." 

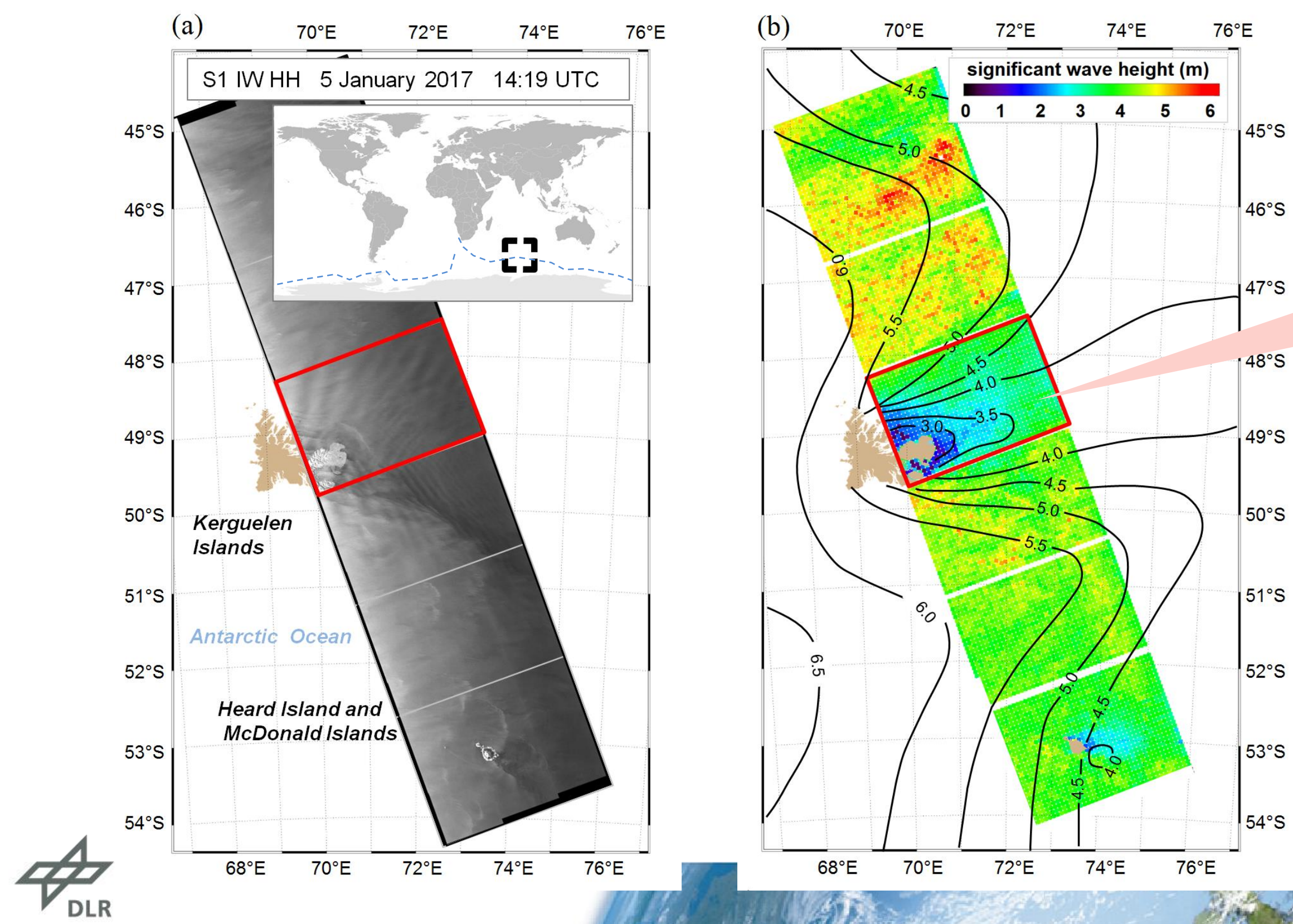

Arctic Sea, 05.01.2017

Processed in NRT And send to research vessel "Akademik Treshnikov" on Antarctic Circumnavigation

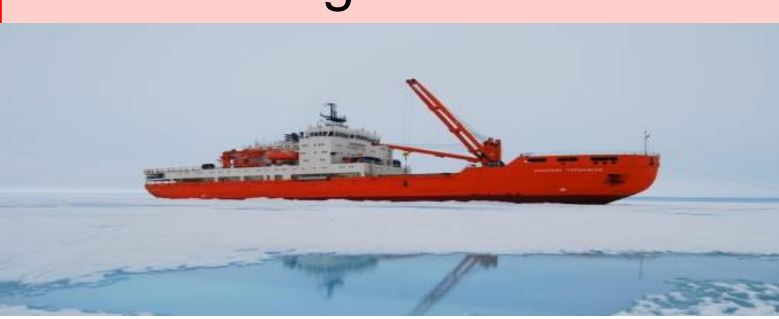


Total Significant Wave Height | Black Sea storm 20-23.04.2017 I SENTINEL -1 SAR C-band IW mode | processing mesh 6km×6km

$\downarrow$ descending path 03:00 UTC

2017-04-20

Modelled storm peak

$\sim 90 \mathrm{~km}$ northerly

\section{7-04-21}

114-m long cargo vessel with 12 crew sinks

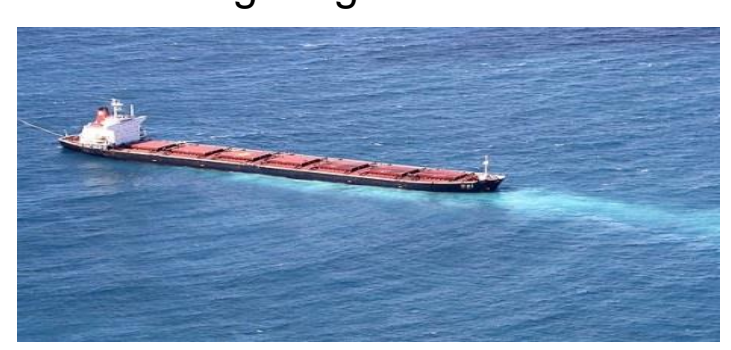

Raging Black Sea storm splits cargo ship in half

2017-04-22

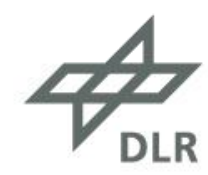

ascending path 16:00 UTC \
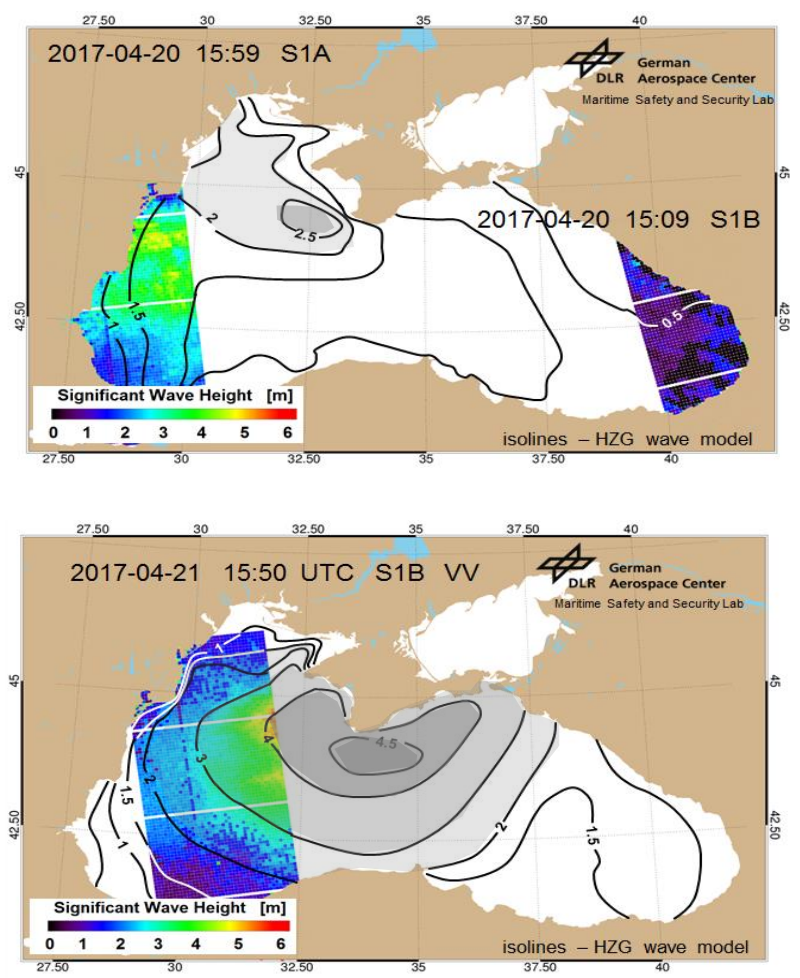

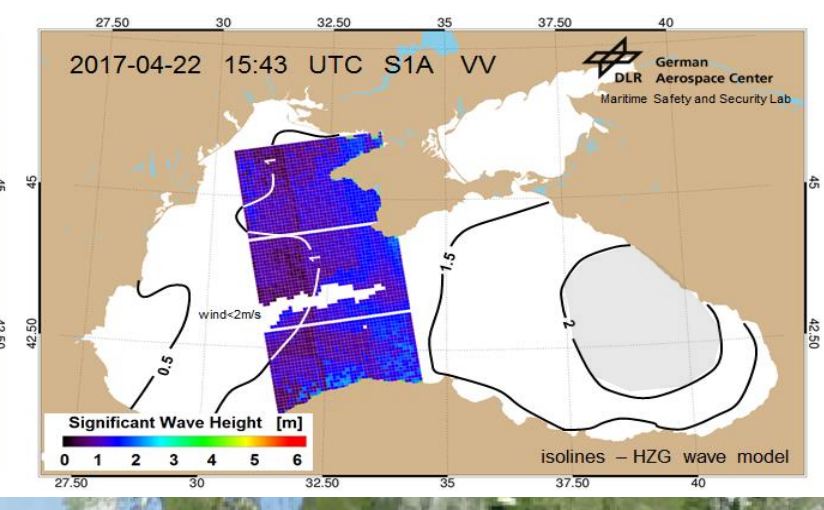

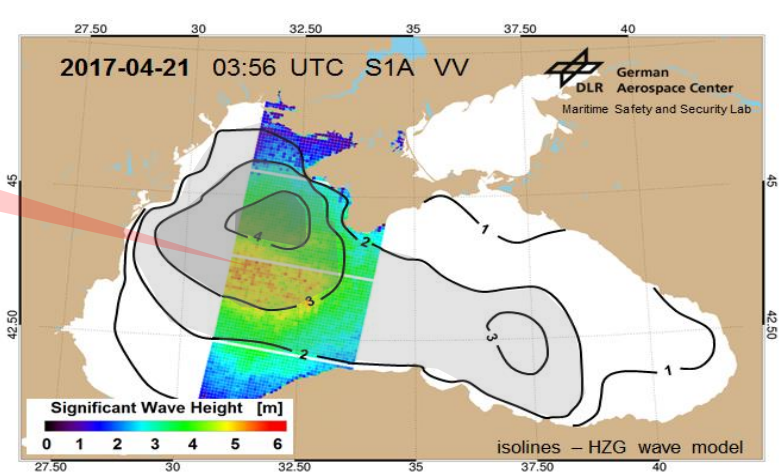

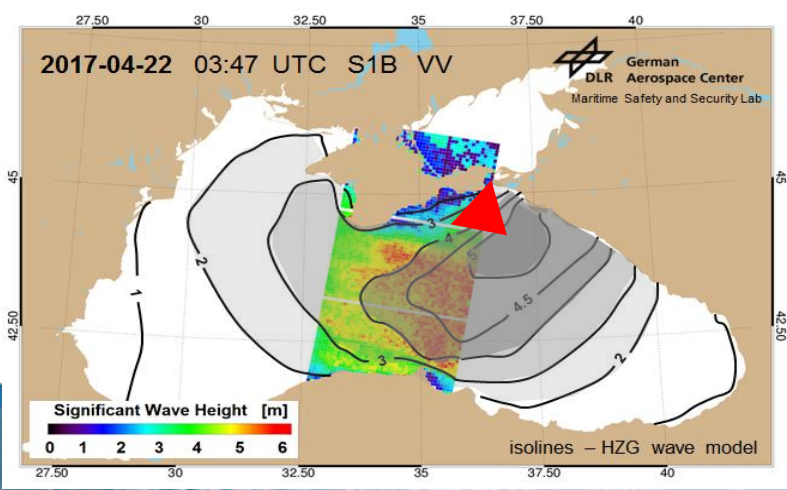


1. Concept and Examples

2. Background

3. Model Functions, Tuning

4. NRT implementation

5. Outllook

4 
1. Basic Research - Functions \& Algorithms

SAR Imaging Mechanism: Geophysical Model Function (GMF): development and adoption

- mathematic investigations

- for practical applications

2. Software Development - Prototype \& NRT Processors

- implementation of GMF into Processors (SSP) prototype

- implementation of SSP into processing chain for NRT cervices,

3. Processing and Results Analysis - What do we learn?

Forecasts improvement and geophysics

- statistics, local distributions

- extreme events

- assessments, danger localization, follow up and validation of forecast models (e.g. DWD)

International Space Station 1998
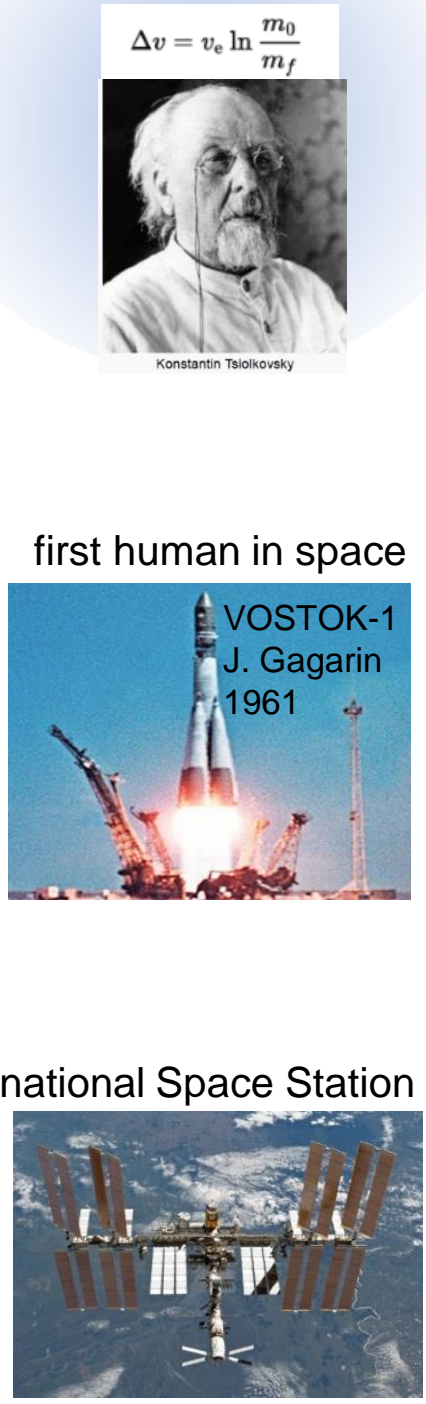

first human in space

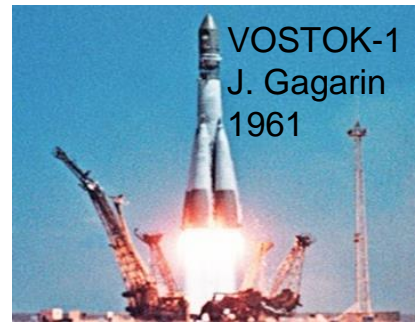




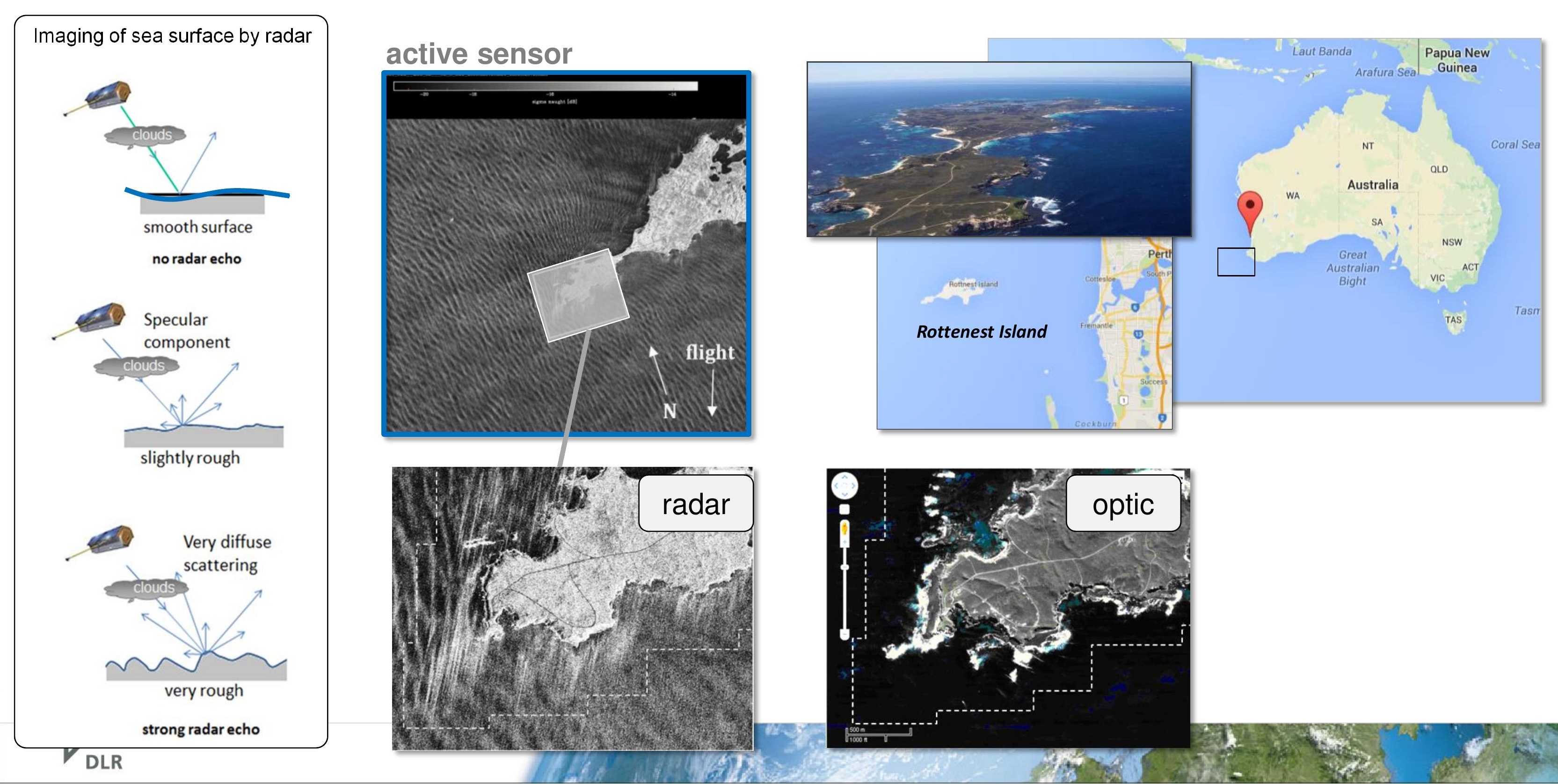

\subsection{Satellite Radar Imagery}

maging of sea surface by radar

2

smooth surface

clouds

slightly rough

DLR 
$\mathrm{Hs} \sim 0.5 \mathrm{~m}$
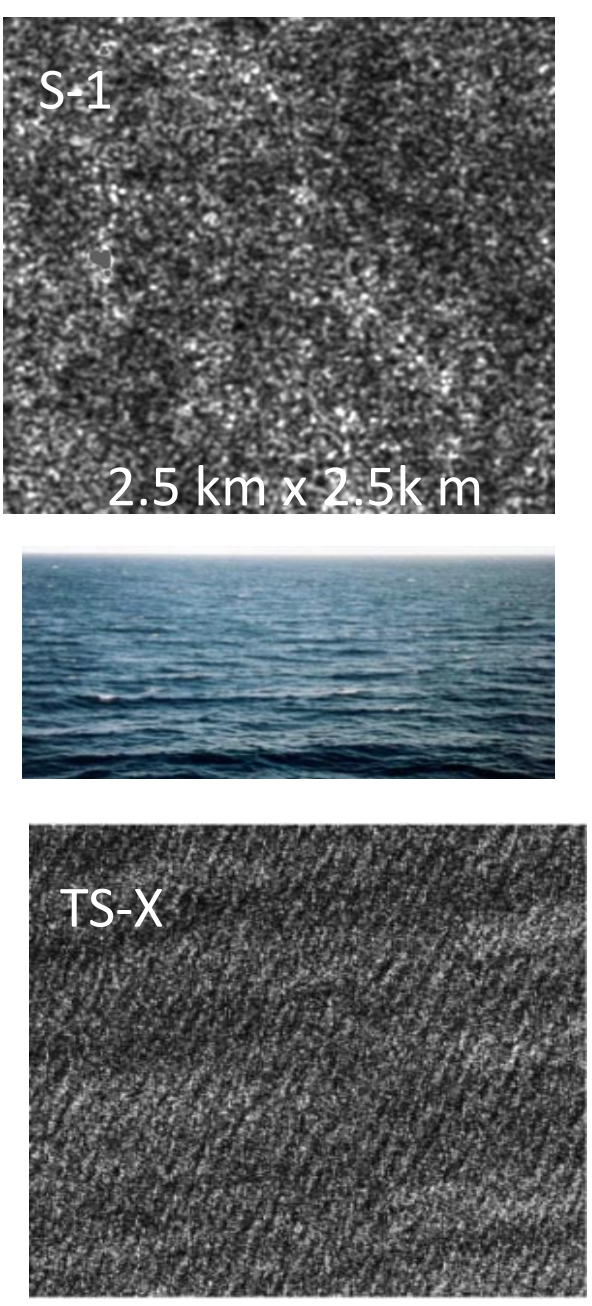

4 calm (swell)
$\mathrm{Hs} \sim 4 \mathrm{~m}$
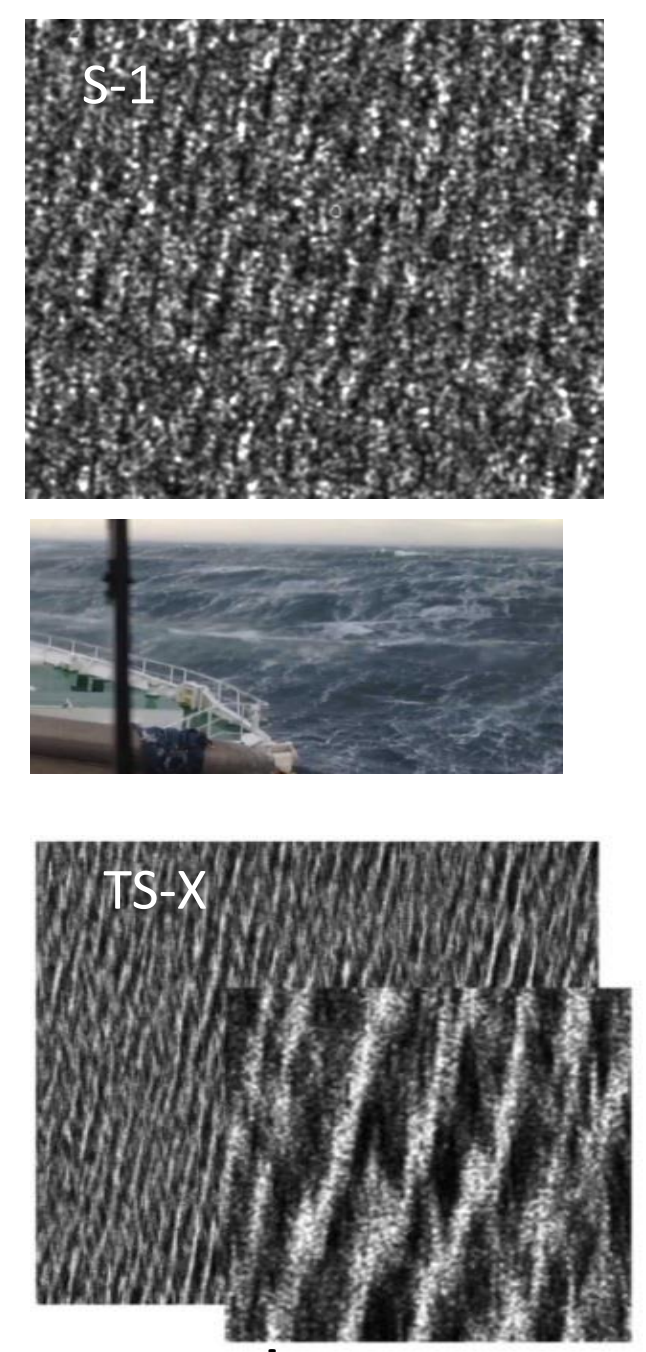

moderate
$\mathrm{Hs} \sim 7 \mathrm{~m}$
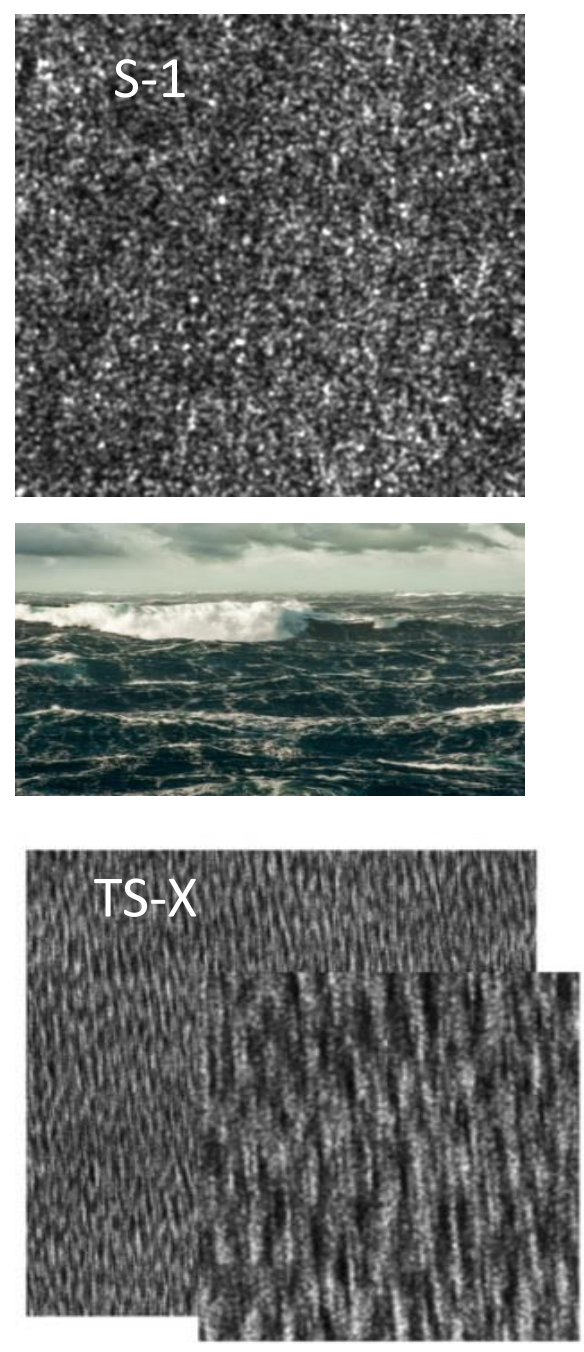

SENTINEL S-1 IW VV 10m Pixel, C-band

TerraSAR-X StripMap VV 1.25m Pixel, X-band

\section{Principle \\ wind and sea state estimation}

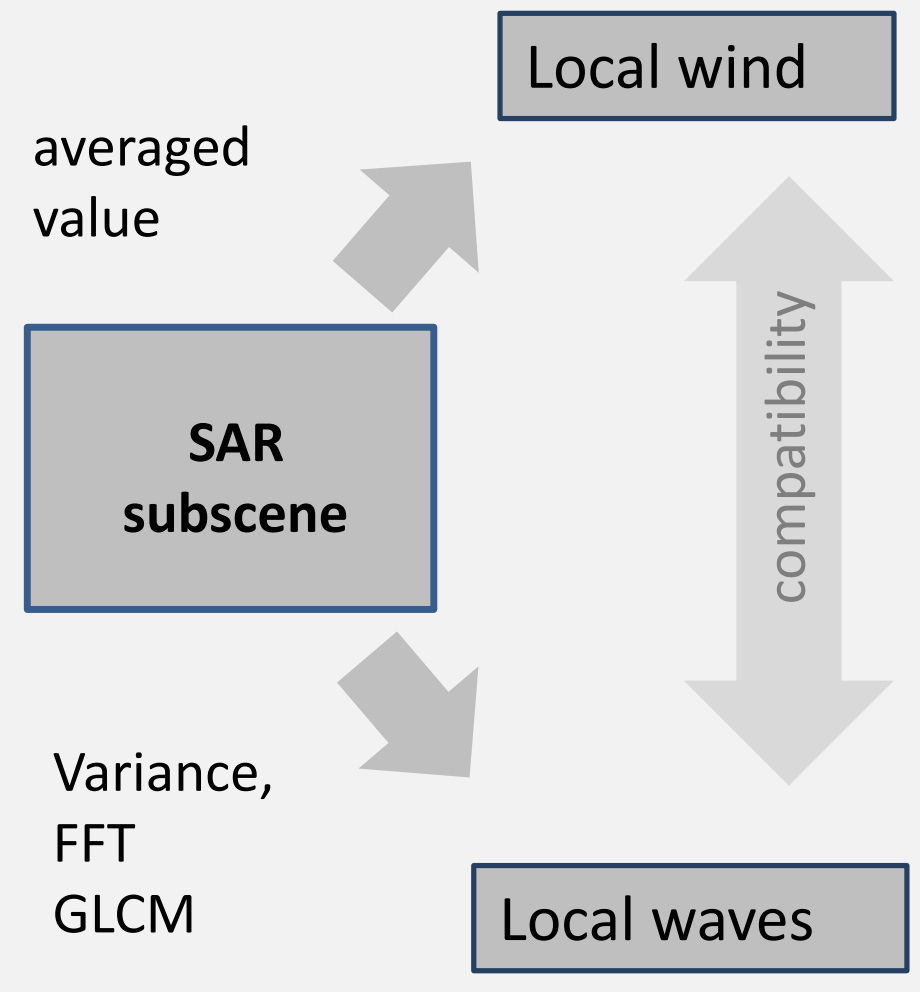


Artefacts in SAR image impact spectra
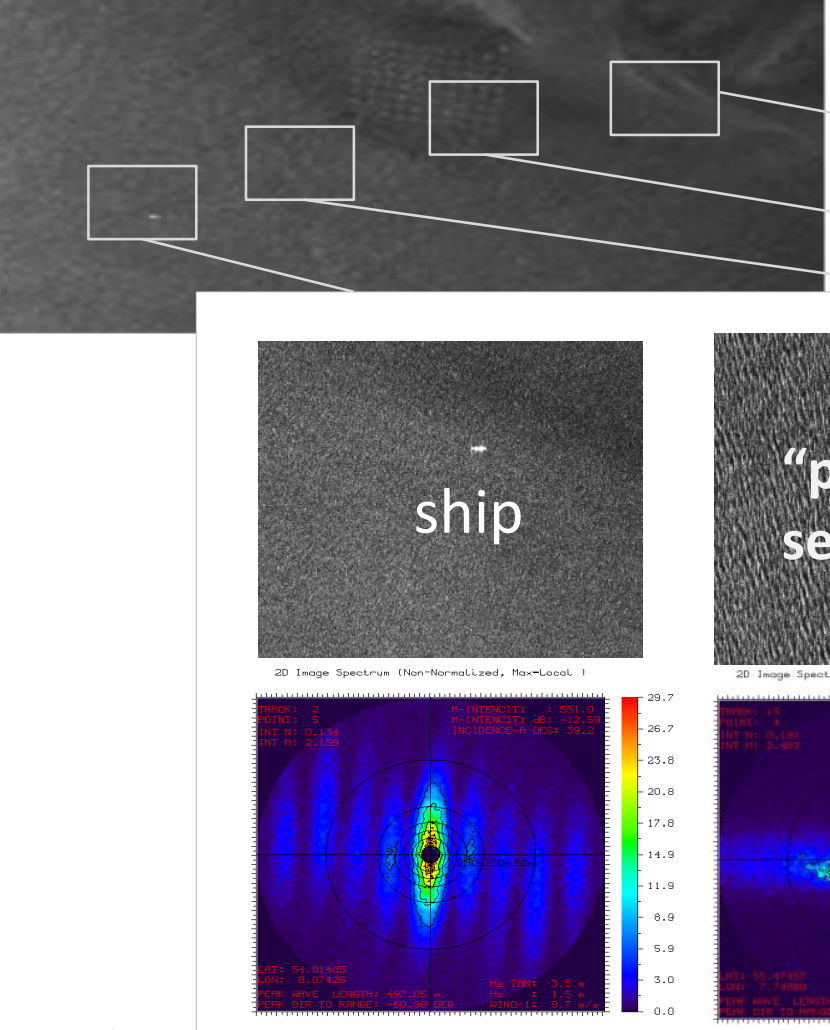

\section{Task №1 - removing artefacts before analysis}

- Sand banks

- Wave breaking

- Ships, Buoys, Wind farms

- Current fronts, ship wakes

Without pre-filtering estimated $\mathrm{Hs}$

can > 10 times overestimate real value
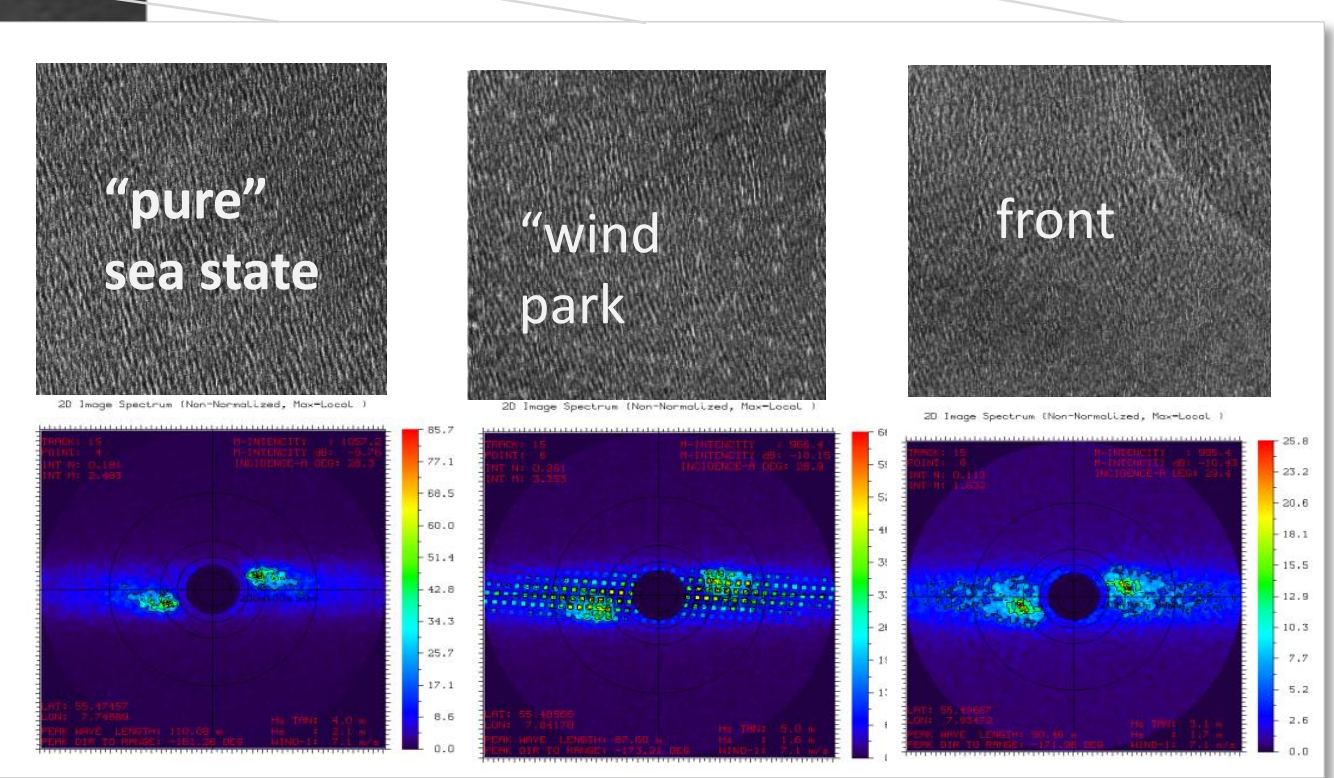

\section{STEPS}

- Removing before analysis - Function correction terms - Control results

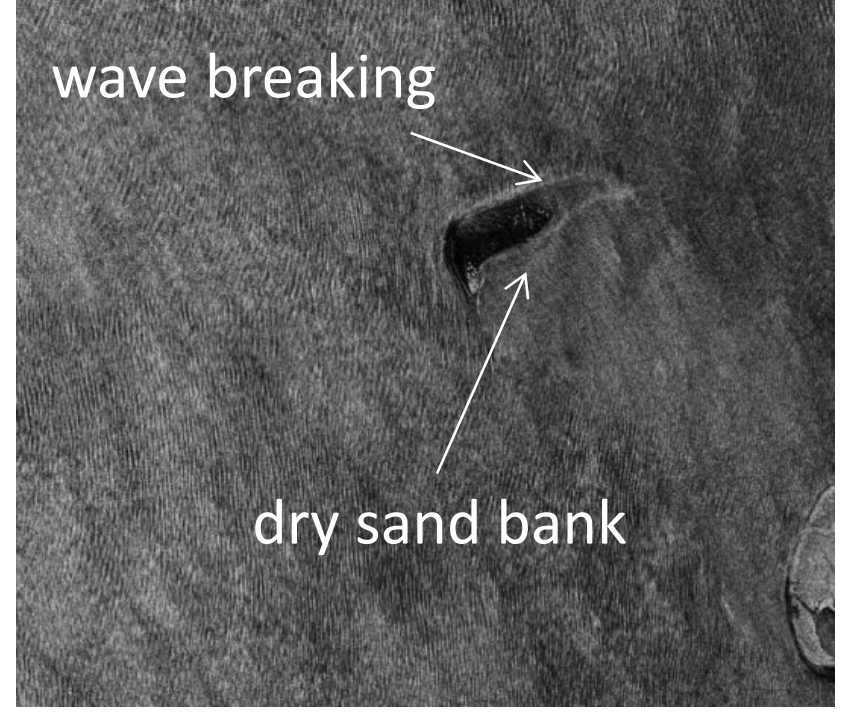


1. Concept and Examples

2. Backoround

3. Model Functions, Tuning

4. NRT implementation

5. Outllook 
3.1. Empirical Function and Parameter (SAR features)

Function: linear regression

$$
P_{i}=\sum_{n=0}^{N} A_{n} S_{n}
$$

\section{SAR features type}

1. Subscene properties and statistics

2. Geophysical

3. GLCM (grey level co-occurrence matrix)

4. Spectral-A
Solution: quadratic minimization using SVD (singular value decomposition) - optimal solution for a linear system

\section{Parameters first order}

NRCS, Norm.-variance, skewness, kurtosis,

+ 5 additional parameters (will be published later)

Wind

GLCM-mean, variance, entropy, correlation, homogeneity, contrast, dissimilarity, energy

using spectral bins for different wavelengths

Goda-parameter, Longuet-Higgins-parameter,

+ 5 additional parameters (will be published later)

\section{Spectral-B}

20 parameter by using orthonormal functions, cutoff by ACF (autocorrelation function) 


\section{Linear regression Empirical Model Function (EMF) bases on parameters}

- Image spectral parameters (20 par.)

- Local wind information, variance

- GLCM (Grey Level Co-Ccurrance Matrix) parameters (Entropy, Homogeneity, Contrast, Dissimilarity, etc.)

$$
W=a_{0}+\sum_{i=1}^{n_{s}} a_{i} s_{i}
$$

Model Function tuning - combination of spectral and Image feature analysis + filters

- tuning by minimizing root mean squared error RMSE

- number of used features improve results

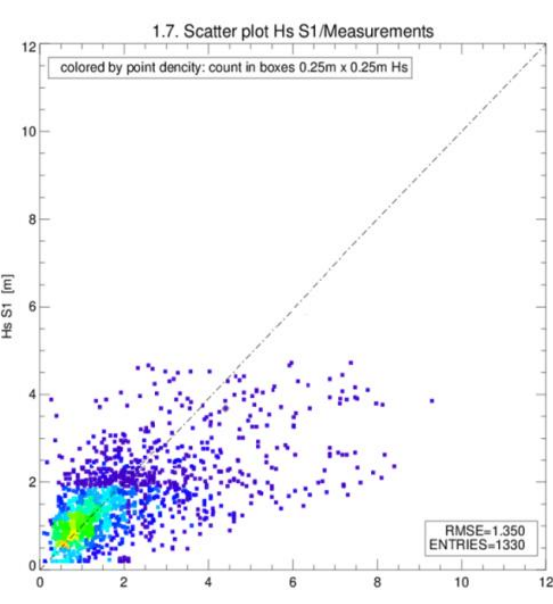

Tuning using

local wind $\mathrm{U}_{10}$ only

(optimal RMSE $=1.35 \mathrm{~m}$ )

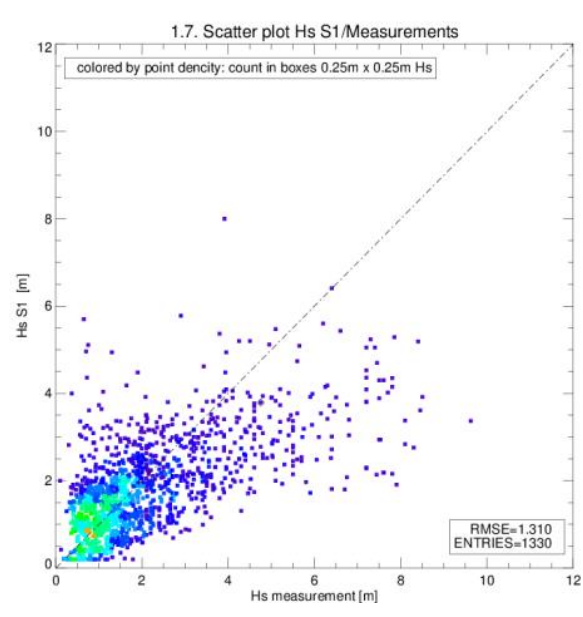

Tuning using

integrated spectrum energy only (optimal RMSE $=1.31 \mathrm{~m}$ )
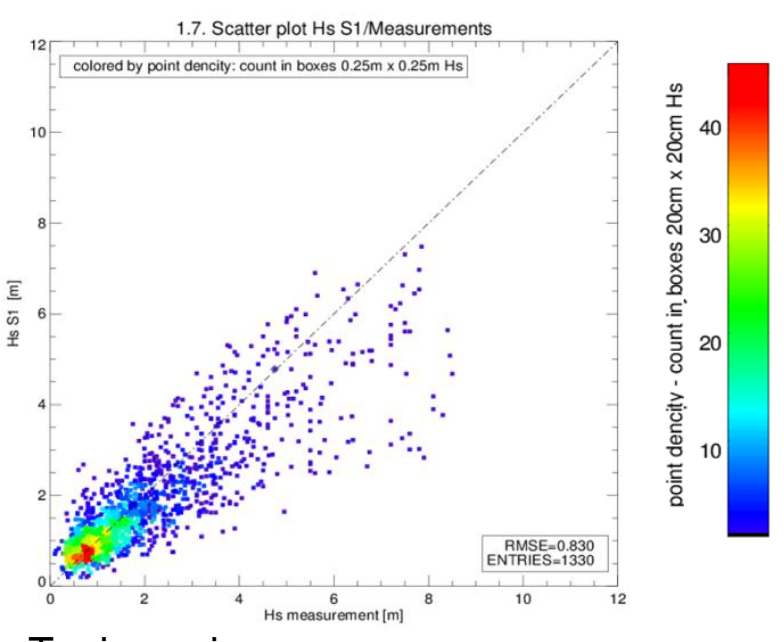

Tuning using:

- integrated image spectrum energy

- local wind $U_{10}$ - spectral parameters - GLCM parameters

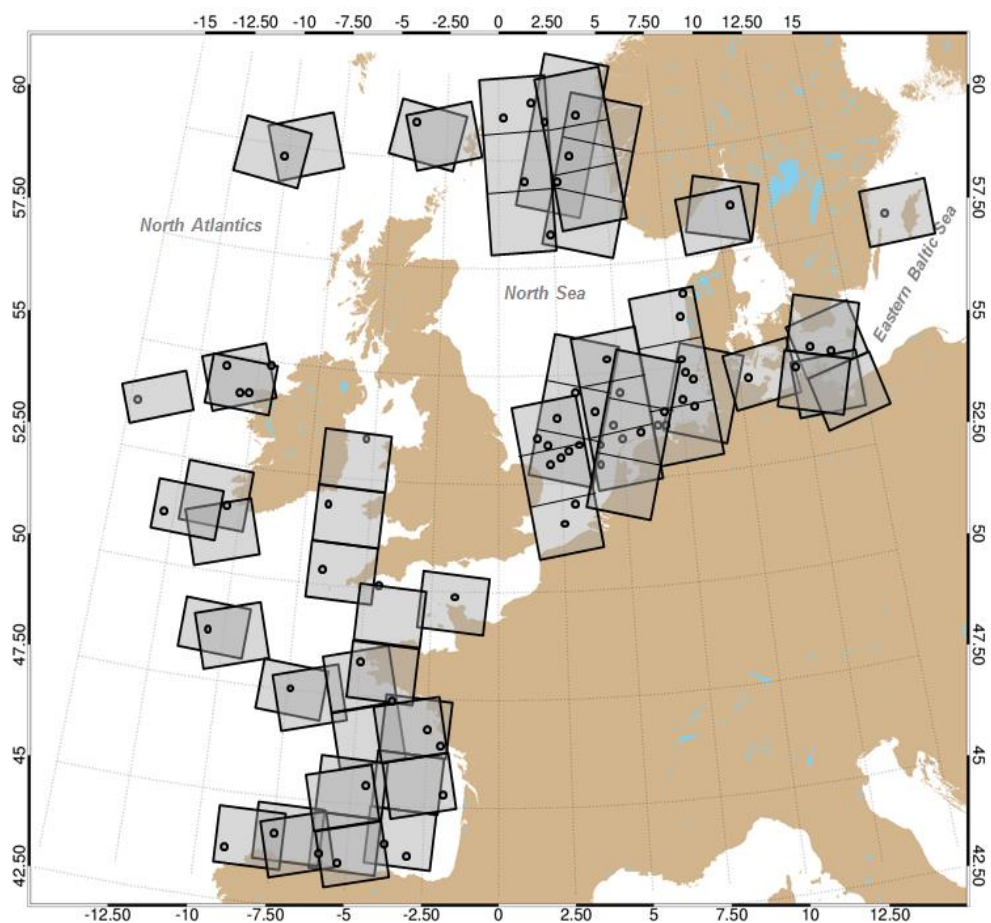

Example for collocations of individual S1 IW images with measurement stations in the

North Sea, Eastern Baltics and North Atlantics used for algorithm tuning and validation. 


\section{Linear regression Empirical Model Function (EMF) bases on parameters}

- Image spectral parameters (20 par.)

- Local wind information, variance

$$
W=a_{0}+\sum_{i=1}^{n_{s}} a_{i} s_{i}
$$

- GLCM (Grey Level Co-Ccurrance Matrix) parameters (Entropy, Homogeneity, Contrast, Dissimilarity, etc.)
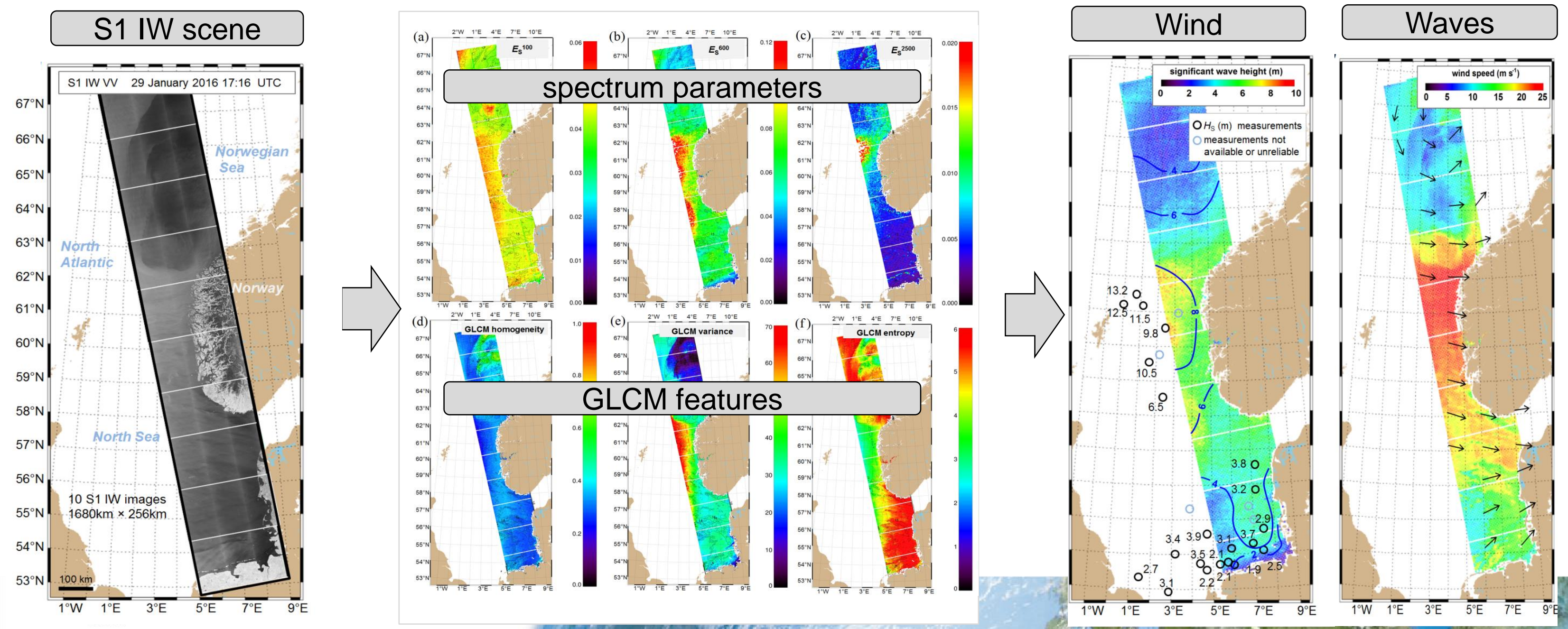
New processor 2020

SAINT Sea State Processor SSP for sea state fields estimation

- New function with new parameters

- New S1 IW acquisition ( 2000) + new validation data -

- New software

CMEMS model results with $\sim 5 \mathrm{~km}$ resolution worldwide (/WW3 $\sim 30 \mathrm{~km}$ resolution)

Collocated SWH distribution, $10 \mathrm{~km}$ raster

- Higher accuracy for SWH + additional parameters

SSP V1-2017 1000 S1-IW

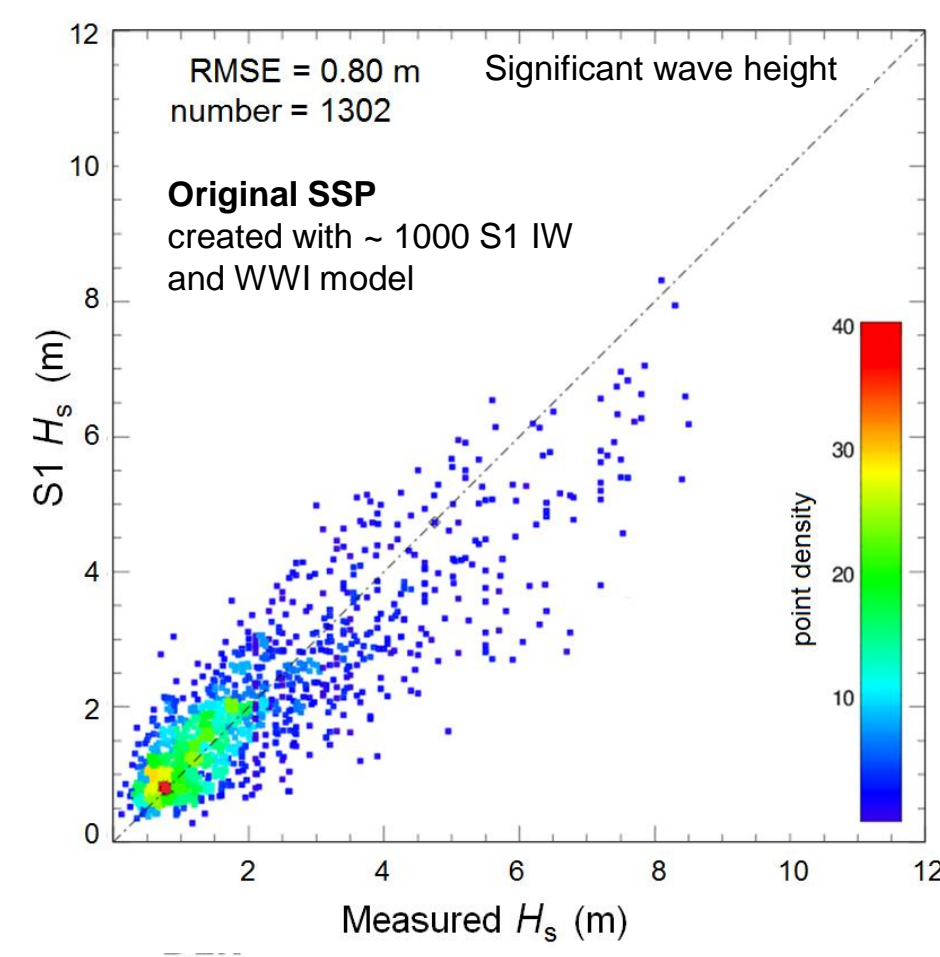

SSP V1-2017 1000 S1-IW

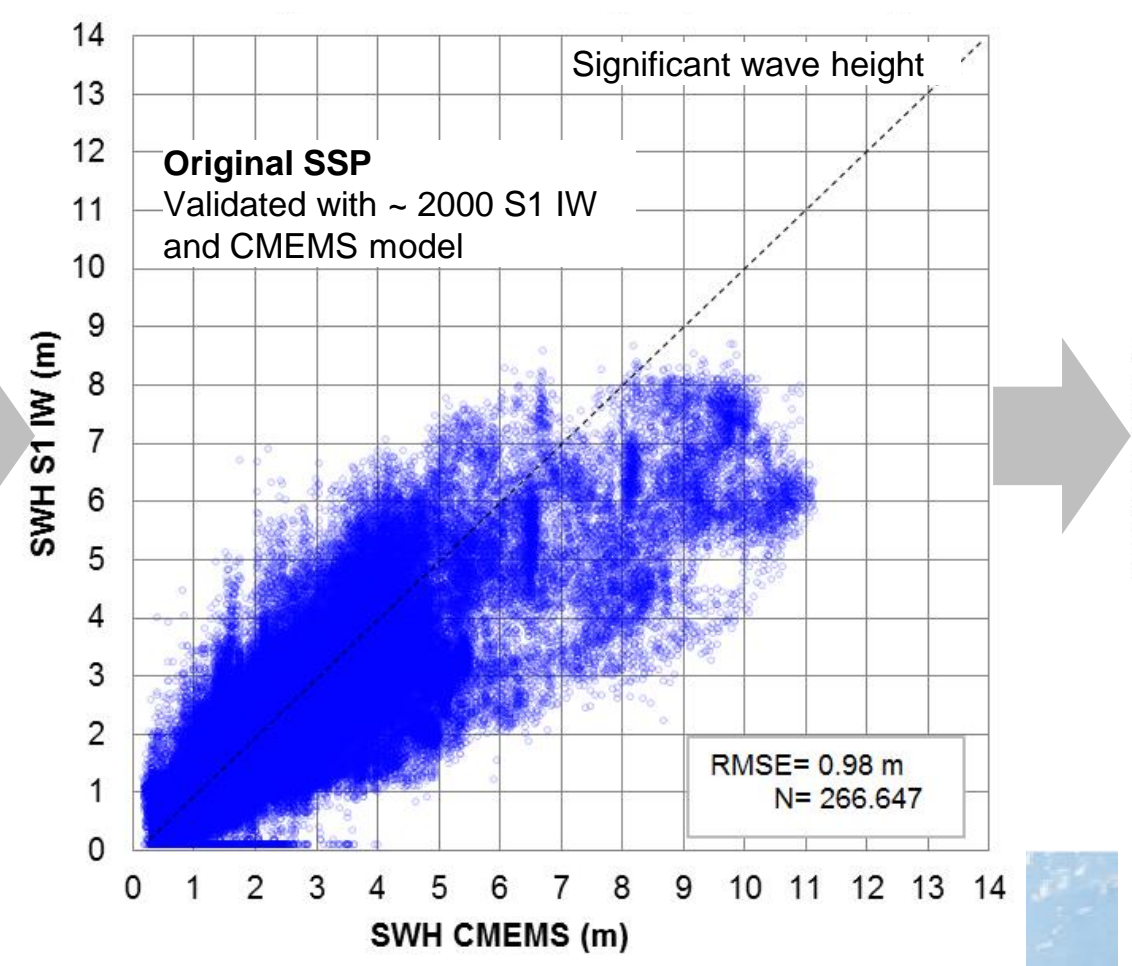

SSP improved 2020

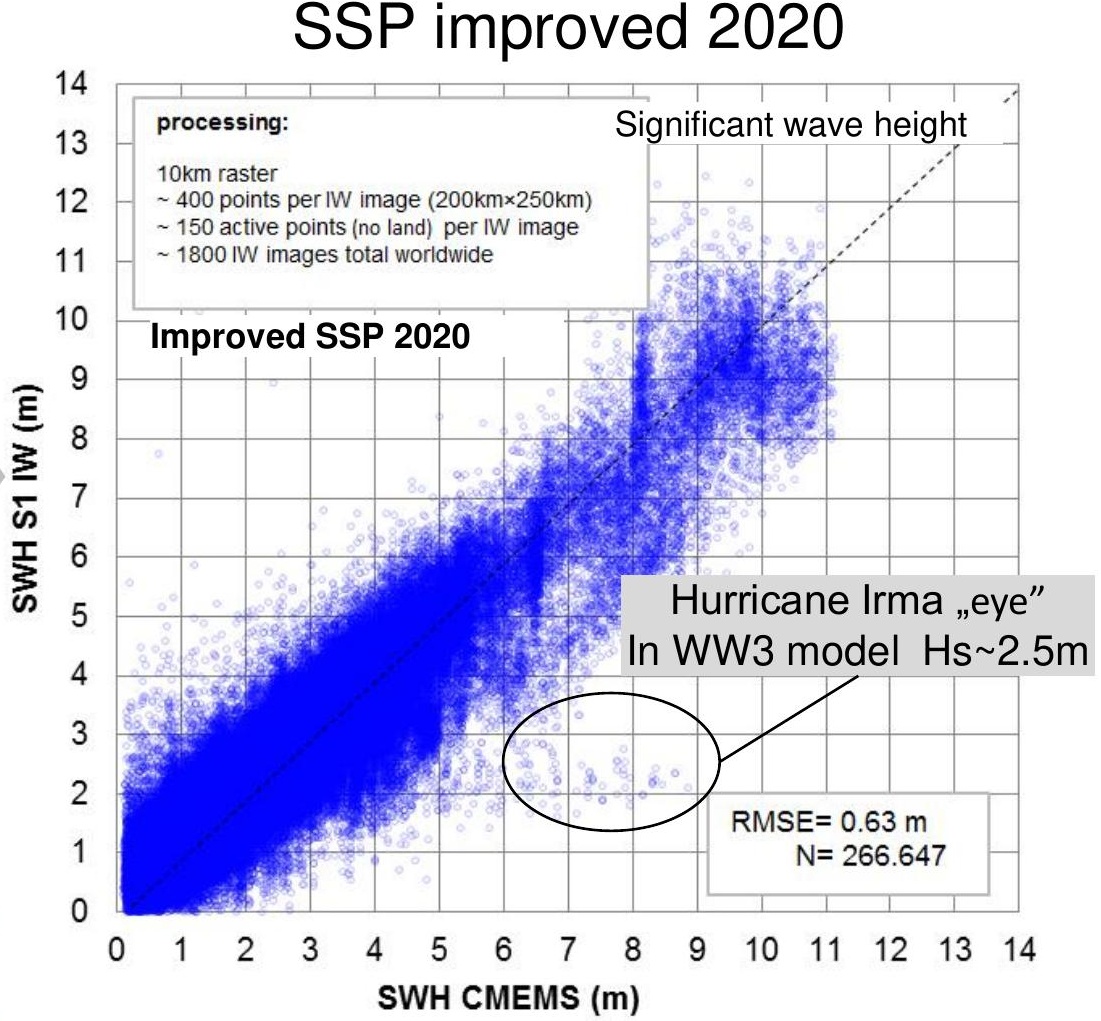




\section{Error distribution SWH S1 IW}

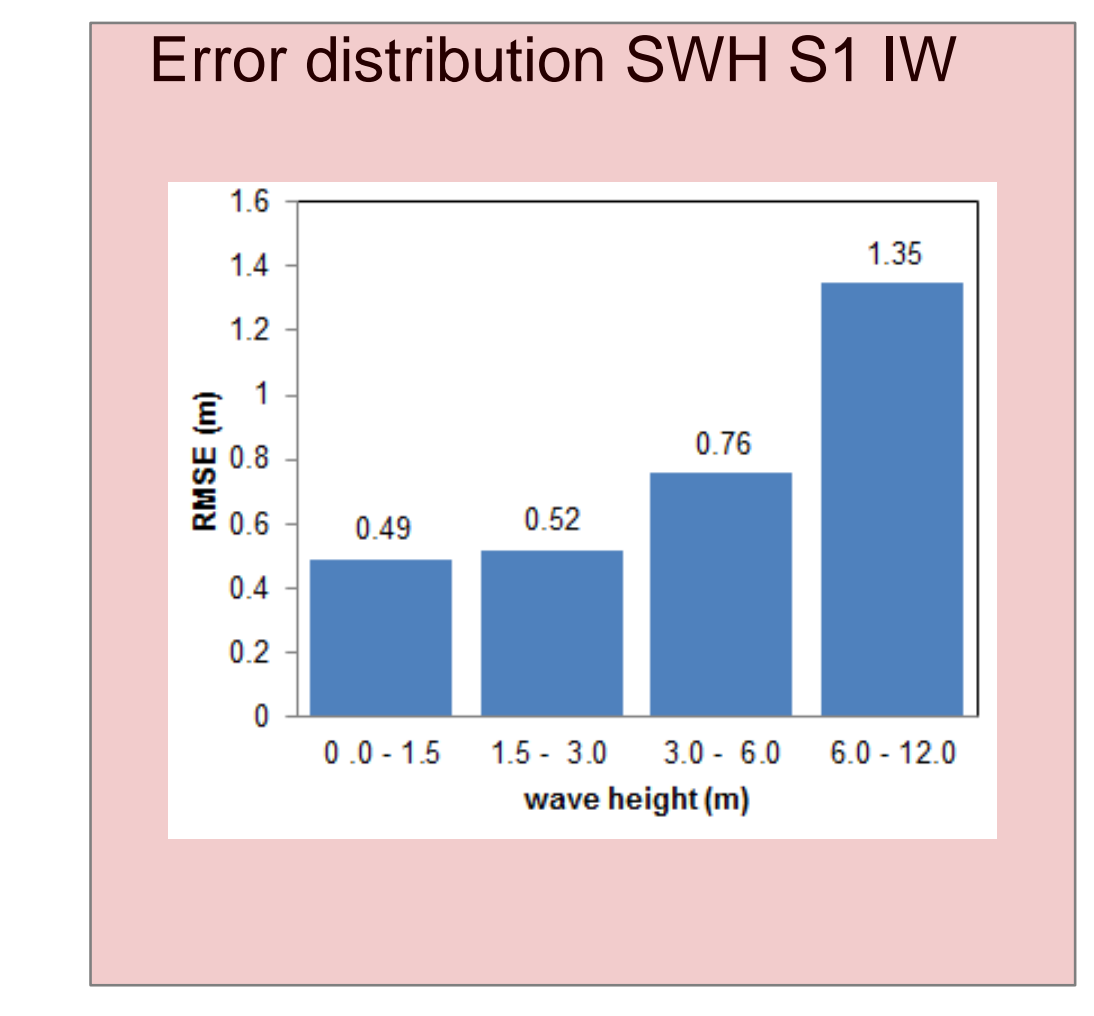

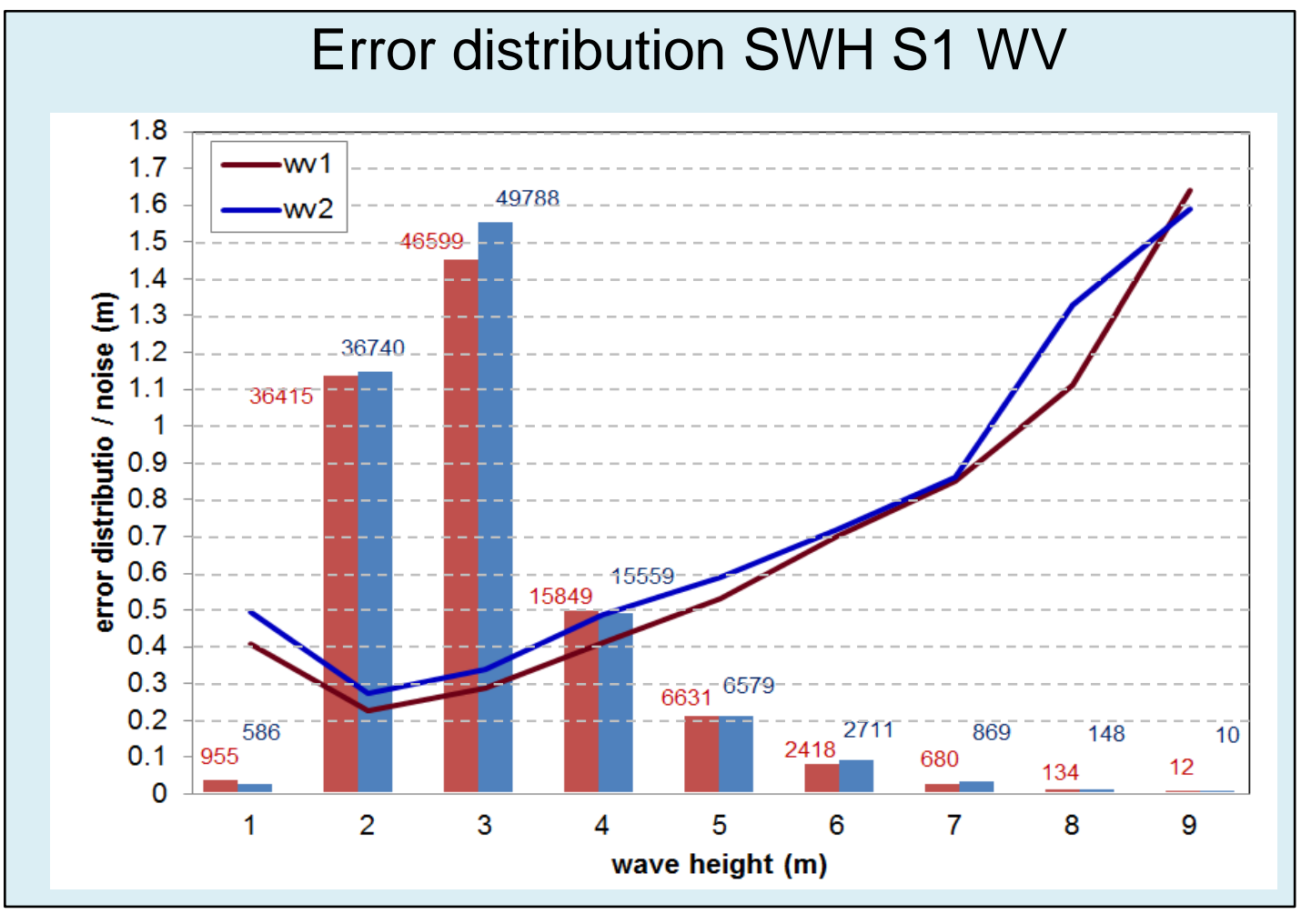

RMSE

Error distribution SWH S1 WV

\begin{tabular}{lcccccccl}
\hline & SWH & Tm0 & Tm1 & Tm2 & Sw1 & Sw2 & Sww & Tw \\
\hline S1 IW & $63 \mathrm{~cm}$ & $1.15 \mathrm{sec}$ & $0.95 \mathrm{sec}$ & $0.79 \mathrm{sec}$ & $0.52 \mathrm{~m}$ & $0.38 \mathrm{~m}$ & $0.73 \mathrm{~m}$ & $0.92 \mathrm{sec}$ \\
\hline S1 WV & $35 \mathrm{~cm}$ & $0.64 \mathrm{sec}$ & $0.52 \mathrm{sec}$ & $0.53 \mathrm{sec}$ & $0.42 \mathrm{~m}$ & $0.35 \mathrm{~m}$ & $0.41 \mathrm{~m}$ & $0.65 \mathrm{sec}$ \\
\hline
\end{tabular}

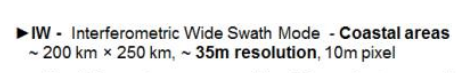

- WV. Wave Moded- Ocean
$\sim 20 \mathrm{~km} \times 20 \mathrm{~km}$ Vignette each $100 \mathrm{~km}, \sim 9 \mathrm{~m}$ resolution $\sim 5 \mathrm{~m}$ pixel
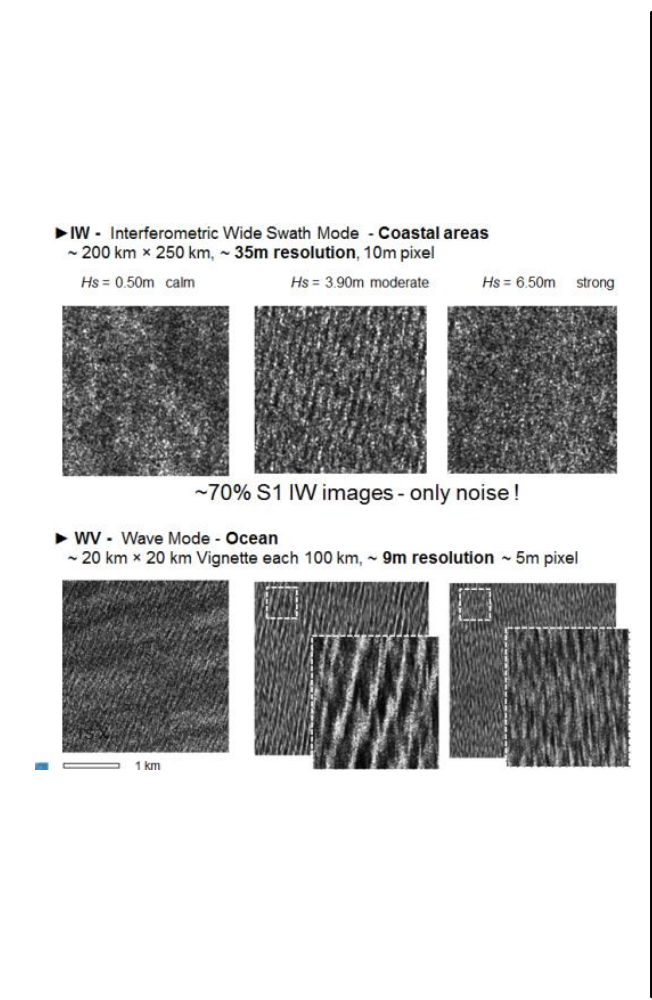
1. Concept and Examples

2. Background

3. Model Functions, Tuning

4. NRT implementation

5. Outlook 


\subsection{Sea State Processor for SENTINEL-1 and TerraSAR-X at Ground Station NZ}
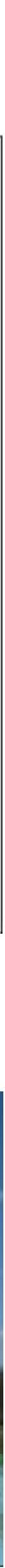
4.2. Acquisitions for a location S1 IW

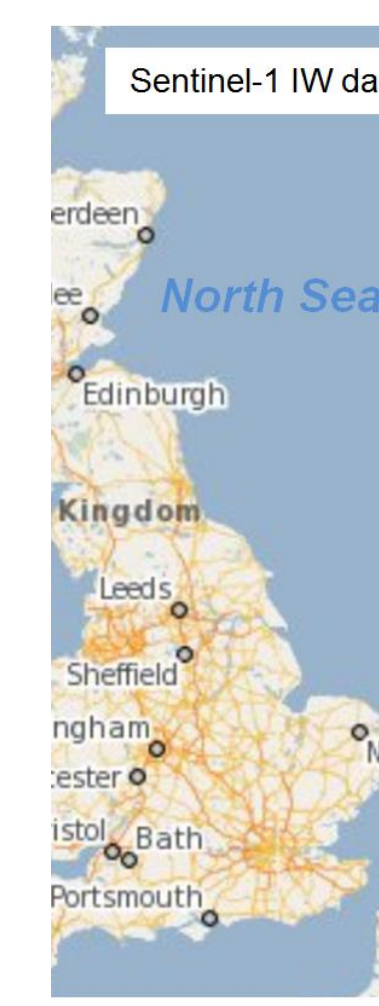

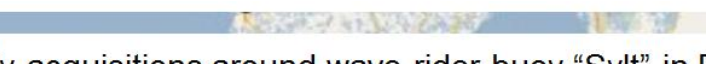
February 2018
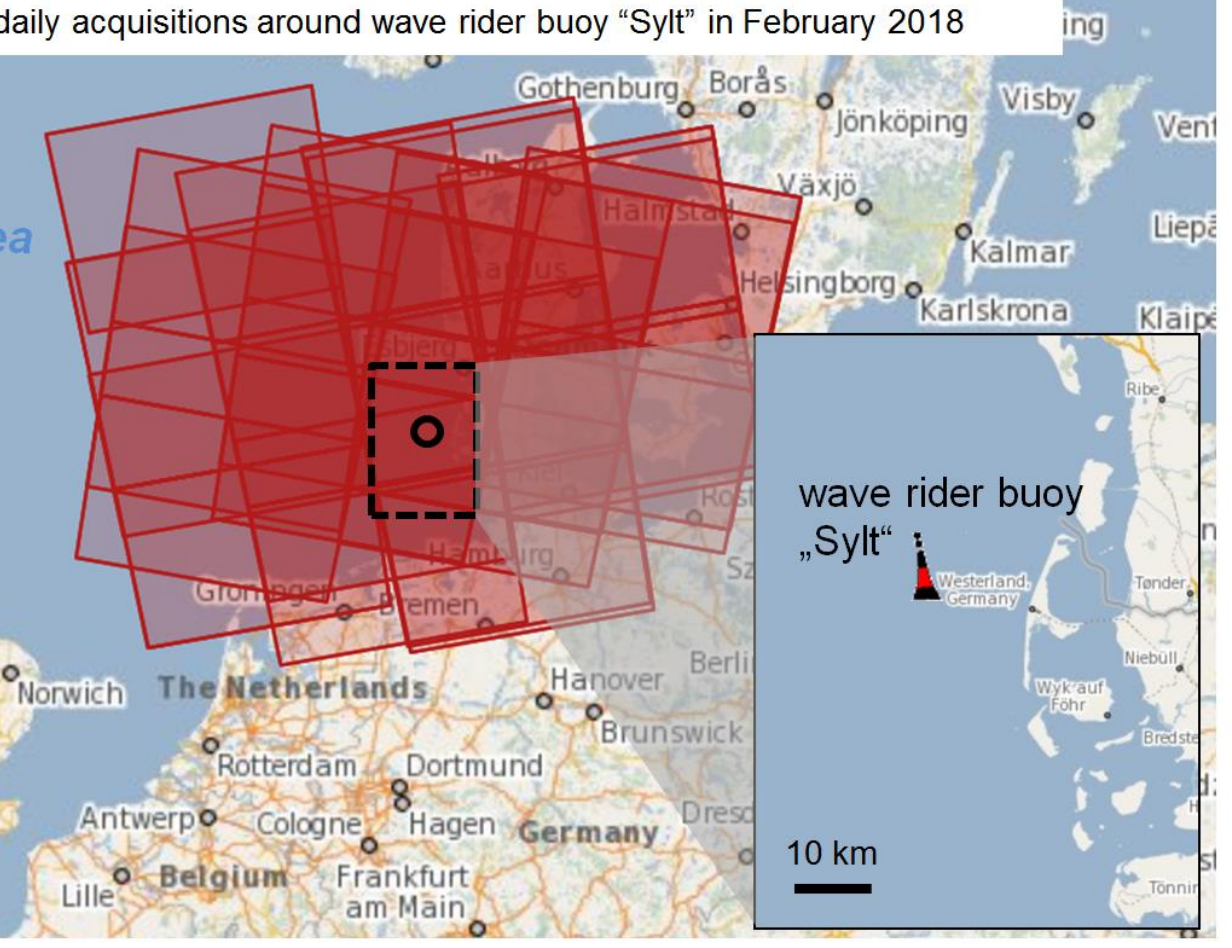

\section{February 2018}

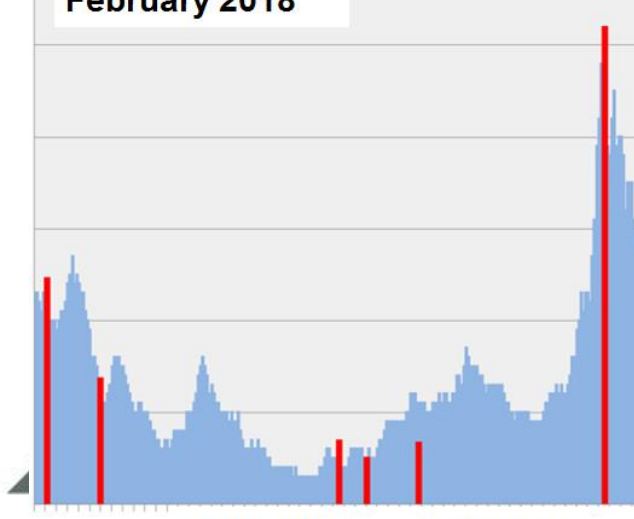

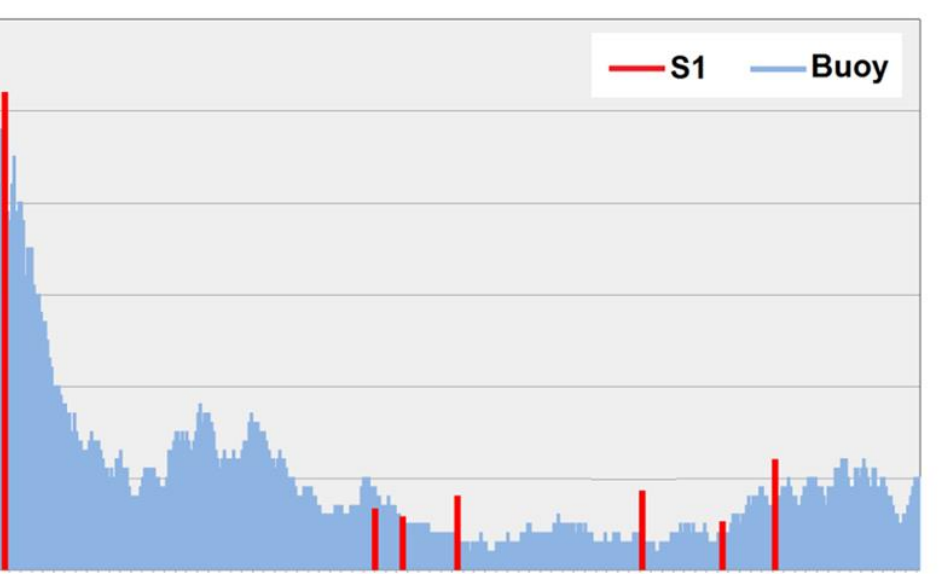

$13 \mathrm{Feb} 2018 \quad 17 \mathrm{Feb} 2018 \quad 21 \mathrm{Feb} 2018 \quad 26$ Feb 2018
2018-03-28 05:41:27

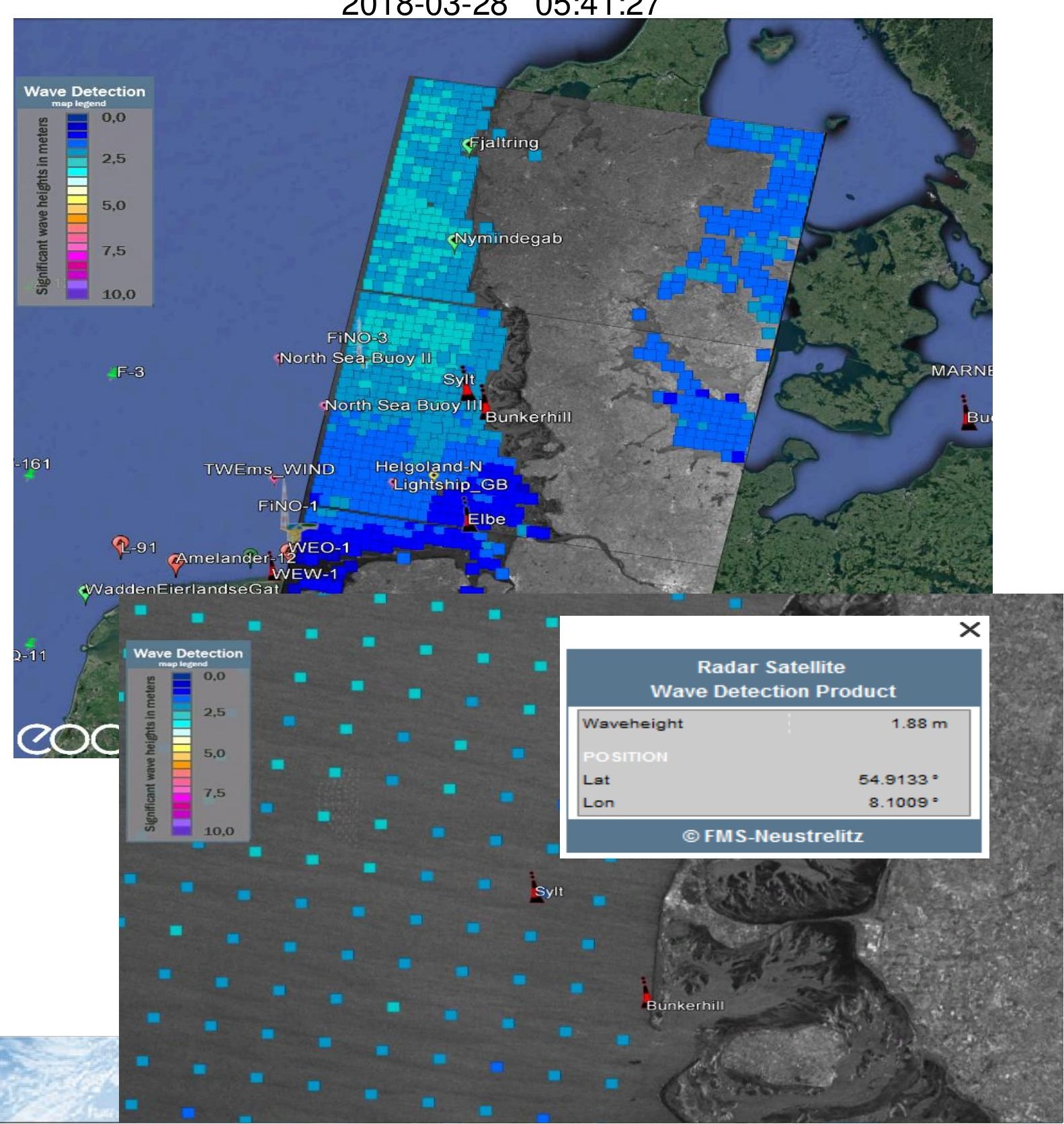

5 Feb 2018 


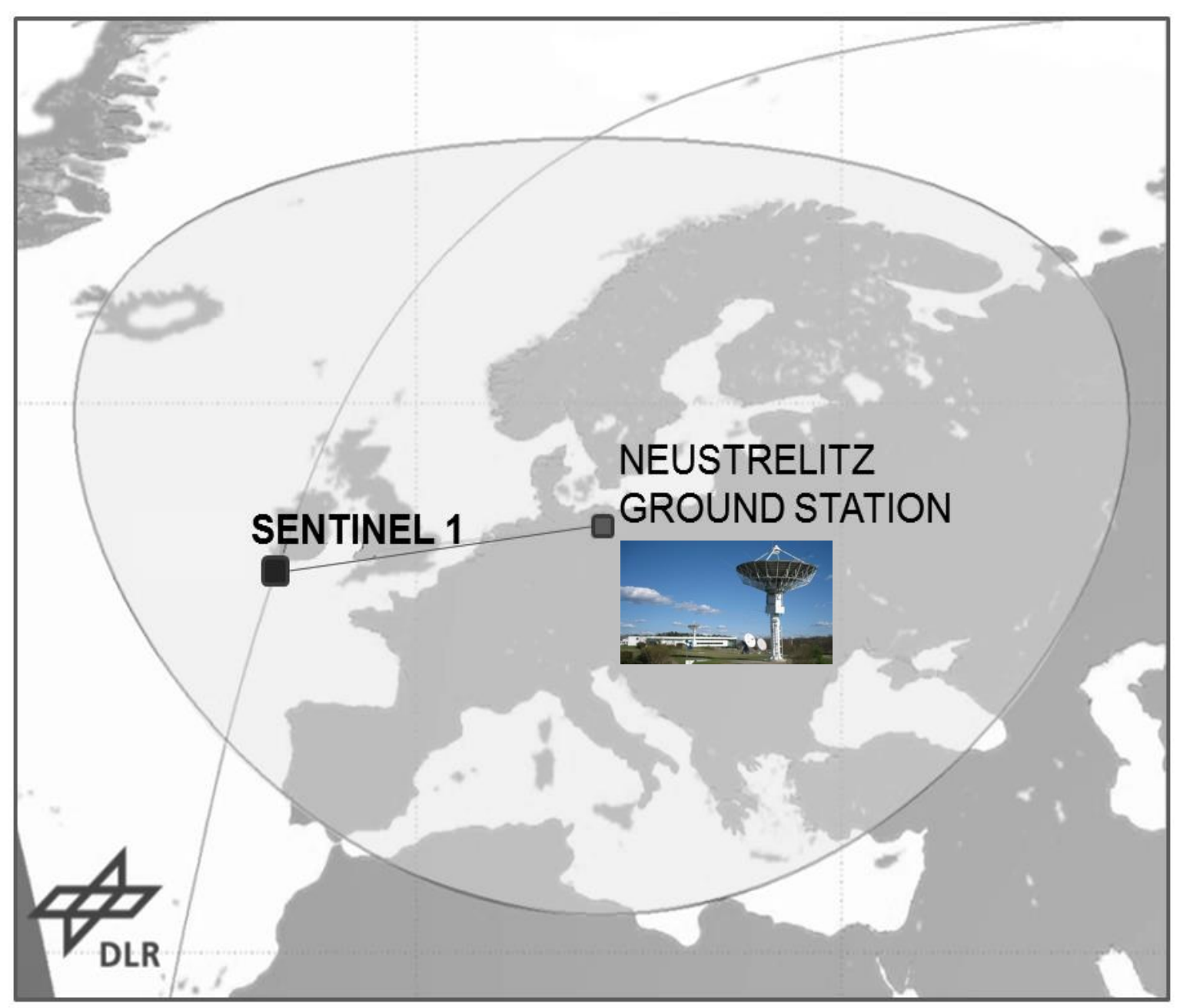

Ground Station Neustrelitz, acquisition circle for Sentinel-1, 5 degree elevation. Inside of this area the data can be transferred from satellites to ground station directly after acquisition, without delay, for NRT processing. 
1. Concept and Examples

2. Background

3. Model Functions, Tuning

4. NRT implementation

5. Outlook

$\oiint_{\mathrm{DLR}}$ 
Sequences of 12 S1-IW images, North Atlantic with Hs of $\sim 9 \mathrm{~m}$ coverage $250 \mathrm{~km} \times 2200 \mathrm{~km}$, Raster $3 \mathrm{~km}$ (60 $\times 80=4800$ subscenes/image $)$.
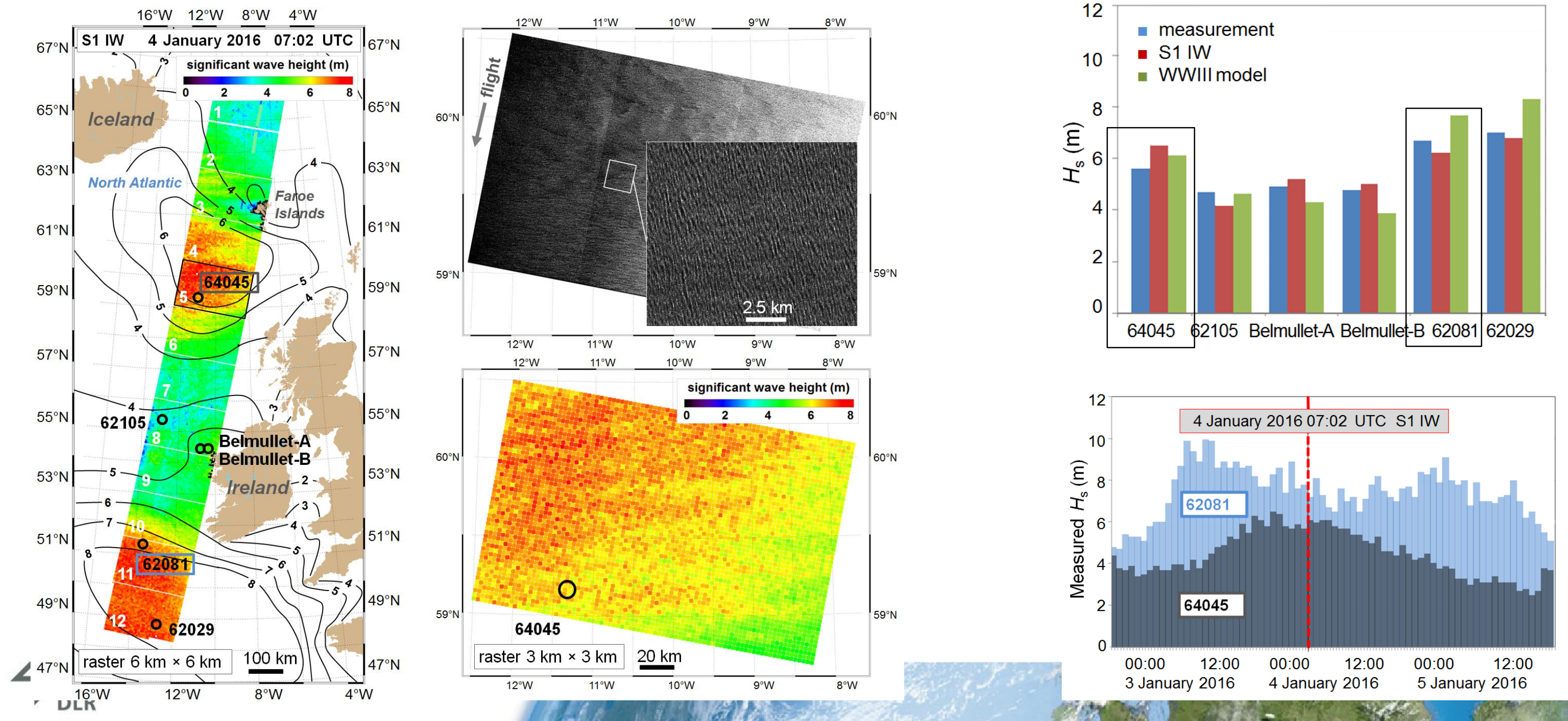